OPEN ACCESS

Edited by:

Xuan (Anna) Li,

University of Maryland, College Park,

United States

Reviewed by:

Mathieu Wolff,

Centre National de la Recherche Scientifique (CNRS), France

Michael M. Halassa,

Massachusetts Institute of

Technology, United States

*Correspondence:

Robert G. Mair

robert.mair@unh.edu

Specialty section:

This article was submitted to Motivation and Reward,

a section of the journal

Frontiers in Behavioral Neuroscience

Received: 15 December 2020 Accepted: 08 March 2021 Published: 08 April 2021

Citation:

Mair RG, Francoeur MJ and

Gibson BM (2021) Central

Thalamic-Medial Prefrontal Control of Adaptive Responding in the Rat:

Many Players in the Chamber.

Front. Behav. Neurosci. 15:642204. doi: 10.3389/fnbeh.2021.642204

\section{Central Thalamic-Medial Prefrontal Control of Adaptive Responding in the Rat: Many Players in the Chamber}

\author{
Robert G. Mair ${ }^{1 *}$, Miranda J. Francoeur ${ }^{1,2}$ and Brett M. Gibson ${ }^{1}$ \\ ${ }^{1}$ Department of Psychology, University of New Hampshire, Durham, NH, United States, ${ }^{2}$ Neural Engineering and Translation \\ Lab, University of California, San Diego, San Diego, CA, United States
}

The medial prefrontal cortex (mPFC) has robust afferent and efferent connections with multiple nuclei clustered in the central thalamus. These nuclei are elements in largescale networks linking mPFC with the hippocampus, basal ganglia, amygdala, other cortical areas, and visceral and arousal systems in the brainstem that give rise to adaptive goal-directed behavior. Lesions of the mediodorsal nucleus (MD), the main source of thalamic input to middle layers of PFC, have limited effects on delayed conditional discriminations, like DMTP and DNMTP, that depend on MPFC. Recent evidence suggests that MD sustains and amplifies neuronal responses in mPFC that represent salient task-related information and is important for detecting and encoding contingencies between actions and their consequences. Lesions of rostral intralaminar (rIL) and ventromedial (VM) nuclei produce delay-independent impairments of egocentric DMTP and DNMTP that resemble effects of mPFC lesions on response speed and accuracy: results consistent with projections of rlL to striatum and VM to motor cortices. The ventral midline and anterior thalamic nuclei affect allocentric spatial cognition and memory consistent with their connections to mPFC and hippocampus. The dorsal midline nuclei spare DMTP and DNMTP. They have been implicated in behavioralstate control and response to salient stimuli in associative learning. mPFC functions are served during DNMTP by discrete populations of neurons with responses related to motor preparation, movements, lever press responses, reinforcement anticipation, reinforcement delivery, and memory delay. Population analyses show that different responses are timed so that they effectively tile the temporal interval from when DNMTP trials are initiated until the end. Event-related responses of MD neurons during DNMTP are predominantly related to movement and reinforcement, information important for DNMTP choice. These responses closely mirror the activity of mPFC neurons with similar responses. Pharmacological inactivation of MD and adjacent rlL affects the expression of diverse action- and outcome-related responses of MPFC neurons. Lesions of MD before training are associated with a shift away from movement-related responses in mPFC important for DNMTP choice. These results suggest that MD has short-term effects on the expression of event-related activity in $\mathrm{MPFC}$ and long-term effects that tune mPFC neurons to respond to task-specific information.

Keywords: central thalamus, prefrontal cortex, reward guided, mediodorsal nucleus of thalamus, intralaminar nuclei of the thalamus, anterior cingulate (ACC), conditional discrimination, midline thalamic nuclei 


\section{INTRODUCTION}

To survive in a dynamic environment organisms must be able to adapt efficiently to changes in conditions, responding in ways that optimize favorable consequences. Behavioral ecologists have demonstrated that foraging animals select among food patches of different quality in a way that maximizes food intake while reducing energy costs (Stephens and Krebs, 1986). Animals often have to simultaneously evaluate other negative and positive factors such as predation risk and mating opportunities (Nonacs, 2001), making seemingly simple decisions more complicated. Rats utilize complex strategies to optimize food acquisition, weighing information about the size and location of food items, exposure, deprivation, circadian time, risk of predation, and possible theft by conspecifics (Whishaw and Dringenberg, 1991; Whishaw et al., 1992). Wood mice in the wild exploit information about food access, experience with the food source, and predation risk in making foraging decisions (NavarroCastilla et al., 2018; Hernández et al., 2019). Many species learn to exploit heterospecific alarm calls to evade potential predators and thus increase their foraging efficiency (Magrath et al., 2015). Eastern gray squirrels shift energy towards vigilance and away from foraging following exposure to red-tailed hawk calls and use subsequent bird chatter as a cue to safety (Lilly et al., 2019). Evolution has equipped organisms with neural mechanisms that allow them to choose a course of action likely to produce favorable consequences based on current goals, past experiences, updated information about actionoutcome contingencies, and sensory evidence. This requires the ability to integrate allocentric information about the external world with egocentric information about the organization and execution of actions, internal state conditions, the anticipation of likely outcomes, and assessment of the actual consequences of behavior.

Medial prefrontal cortex (mPFC) plays a critical role in adaptive goal-directed behavior (Miller and Cohen, 2001; Dalley et al., 2004; Chudasama, 2011; Kesner and Churchwell, 2011; Balleine, 2019). mPFC has robust afferent and efferent connections with multiple thalamic nuclei that are clustered in the central thalamus and are hence referred to as central thalamic nuclei (Figure 1). Central thalamic nuclei are elements in large-scale networks connecting mPFC with the hippocampus, basal ganglia, amygdala, other areas of the neocortex, and visceral and arousal systems in the brainstem that give rise to adaptive goal-directed behavior. They include the paraventricular $(\mathrm{PV})$, paratenial $(\mathrm{PT})$, reuniens $(\mathrm{Re})$, and rhomboid $(\mathrm{Rh})$ midline nuclei; the central medial $(\mathrm{CM})$, central lateral (CL), and paracentral (PC) rostral intralaminar nuclei; the anterior medial (AM) and interanteromedial (IAM) anterior nuclei; and the mediodorsal (MD) and ventromedial (VM) nuclei (Groenewegen, 1988; Sesack et al., 1989; Berendse and Groenewegen, 1991; Ray and Price, 1992; Vertes, 2002, 2004; Hoover and Vertes, 2007). Although early studies focused on $\mathrm{MD}$, the main source of thalamic input to middle layers of $\mathrm{mPFC}$, it is now clear that MD has limited effects on mPFC function and that other central thalamic nuclei contribute importantly to the effects of mPFC on goal-directed behavior (Dalley et al., 2004;

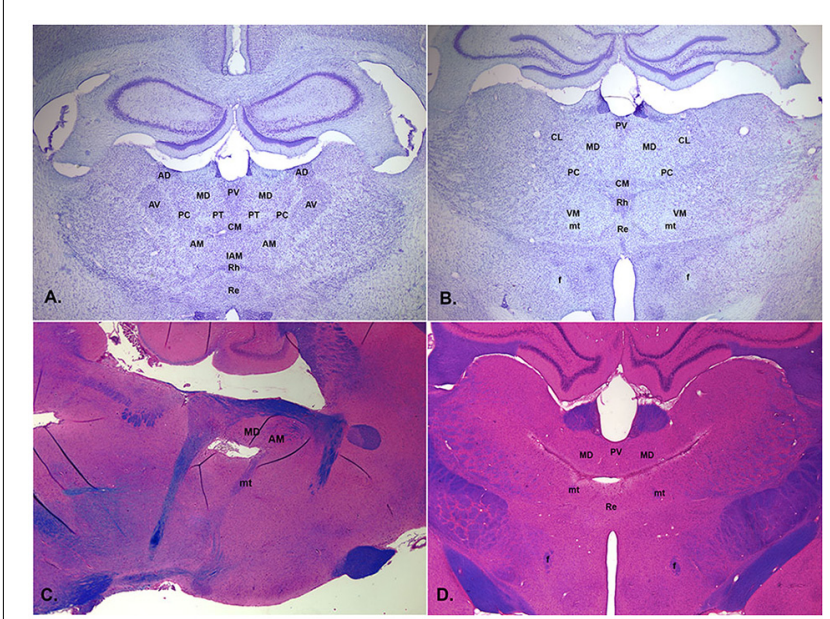

FIGURE 1 | Low magnification photomicrographs of central thalamus showing normal appearance of coronal sections stained with thionin approximately $2.3 \mathrm{~mm}$ (A) and $2.8 \mathrm{~mm}$ (B) posterior to bregma and thalamic lesions from the post-thiamine deficiency (PTD) model of the

Wernicke-Korsakoff syndrome (WKS) stained with luxol blue, hematoxylin, and eosin in the sagittal section about $0.9 \mathrm{~mm}$ off midline $(\mathbf{C}$, tissue from Mair et al., 1988) and in the coronal section about $3.2 \mathrm{~mm}$ posterior to bregma (D, tissue from Mair et al., 1991). PTD treatment produces bilaterally symmetric lesions centered on the mediodorsal (MD) and intralaminar nuclei that tend to spare the anterior nuclei. It is associated with behavioral impairments consistent with the performance of WKS patients on comparative neuropsychological tasks (Mair, 1994). Labeled structures include the MD, centrolateral $(C L)$, paracentral $(P C)$, central medial $(C M)$, reuniens $(R e)$, rhomboid (Rh), ventromedial (VM), anterodorsal (AD), anteromedial (AM), anteroventral (AV), interanteromedial (IAM), and paratenial (PT) nuclei and the fornix $(f)$ and mammillothalamic tract $(\mathrm{mt})$.

Balleine and O'Doherty, 2010; Chudasama, 2011; Euston et al., 2012; Mitchell et al., 2014; Mair et al., 2015; Marton et al., 2018; Parnaudeau et al., 2018; O'Mara and Aggleton, 2019; Wolff and Vann, 2019; McGinty and Otis, 2020).

Vertes et al. (2015) classified a group of nuclei along the thalamic midline as limbic based on prominent afferent and efferent connections with limbic-related structures and evidence that they serve limbic-related functions, including affective behaviors, reward-guided responding, response-related working memory, and behavioral flexibility. These nuclei include PV, PT, $\mathrm{Re}, \mathrm{Rh}$, and CM nuclei and the medial division of MD. PC, $\mathrm{CL}$, and more lateral divisions of $\mathrm{MD}$ are reciprocally connected to more dorsal motor-related areas in anterior cingulate and agranular medial areas of mPFC (Groenewegen, 1988; Berendse and Groenewegen, 1991; Ray and Price, 1992; Vertes, 2002; Hoover and Vertes, 2007). Recent evidence indicates that thalamocortical neurons in MD are strongly excited by the driver and modulatory input from PFC and indirectly influence reciprocally-connected neurons in PFC by enhancing cortical connectivity and regulating neuronal activity (Barbas et al., 1991; Xiao et al., 2009; Schmitt et al., 2017; Collins et al., 2018). The projections of the rostral intralaminar nuclei have thalamocortical and thalamostriatal projections to areas that are interconnected by corticostriatal projections: connections that appear organized to control interactions between $\mathrm{MPFC}$ and the 
basal ganglia and thus selection of goals, actions, and associative stimuli (Berendse and Groenewegen, 1990; Groenewegen and Berendse, 1994; Grillner et al., 2005; Mannella et al., 2016). The intralaminar and midline nuclei receive prominent subcortical inputs from periaqueductal gray, parabrachial nuclei, superior colliculus, hypothalamus, and brainstem nuclei (Krout and Loewy, 2000a,b; Krout et al., 2001, 2002; Bayer et al., 2002). These provide signals related to visceral, nociceptive, orienting, and arousal functions consistent with a role for these nuclei in behavioral state control of mPFC function (Kinomura et al., 1996; Schiff and Purpura, 2002; Mair and Hembrook, 2008).

The ventromedial nucleus (VM) has dense reciprocal connections with the agranular medial cortex and adjacent motor and cingulate areas. Afferent inputs to VM include branches of axons that also innervate $\mathrm{MD}$ and GABAergic projections from the basal ganglia. Thalamocortical neurons in VM have dense widespread projections to layer 1 in agranular medial and adjacent motor and cingulate cortices and less dense projections in parietal and occipital cortices that appear organized to control integrative motor responses (Vertes, 2002; Hoover and Vertes, 2007; Kuramoto et al., 2017; Collins et al., 2018; Sierveritz et al., 2019). The anterior thalamic nuclei receive inputs from the subicular complex of the hippocampal formation and are reciprocally connected to the retrosplenial cortex, an important hub for spatial cognition. The IAM and AM nuclei are also reciprocally connected to anterior cingulate and prelimbic areas of mPFC (Vertes, 2002; Hoover and Vertes, 2007). Lesions of the anterior thalamic nuclei affect allocentric spatial learning tasks (Aggleton and Nelson, 2015; O’Mara and Aggleton, 2019).

Anatomical analyses indicate that rodent $\mathrm{mPFC}$ is homologous to primate anterior cingulate and premotor cortices and lacks an area homologous to primate dlPFC (Preuss, 1995; Uylings et al., 2003; Vogt et al., 2013; Schaeffer et al., 2020). Here we focus on interactions between mPFC and thalamus in rodents to avoid the complexity of factoring in the influence of primate dlPFC on thalamocortical interactions. In this article, we review the two convergent approaches that have been used to elucidate the role of thalamo-prefrontal pathways in generating adaptive responses. The first is to lesion or manipulate the activity of these pathways and study the effects on behavioral measures of adaptive responding. Here we use behavioral measures to examine the influence of different thalamic nuclei on functions supported by mPFC. The second is to record the activity of $\mathrm{mPFC}$ and central thalamic neurons in awake, behaving animals to understand what information is represented and how cortical and thalamic neurons interact during adaptive goal-directed behavior. Here, we emphasize recordings comparing $\mathrm{mPFC}$ with $\mathrm{MD}$, given the lack of data to support comparisons with other central thalamic nuclei. We focus on spatial delayed conditional discriminations: tasks that have received considerable attention in both behavioral and electrophysiological recording studies. These tasks incorporate important features of adaptive goal-directed responding: flexible reward-guided choice, where different responses are reinforced on different trials; conditional discrimination, where a preceding stimulus indicates which response alternative will be reinforced; working memory, where information must be represented briefly in memory; spatial navigation, where behavioral events are distributed topographically; and motor planning, where organisms must organize and execute a series of actions to obtain reinforcement. Lesion studies in rats have provided evidence that pathways connecting $\mathrm{mPFC}$ with the striatum, pallidum, and thalamus are critical for spatial delayed conditional discrimination (Dunnett, 1990; Reading and Dunnett, 1991; Kesner et al., 1996; Mair et al., 1998, 2002; Floresco et al., 1999; Burk and Mair, 2001a; Porter et al., 2001; Bailey and Mair, 2005; Zhang et al., 2005; Sloan et al., 2006).

\section{BEHAVIORAL STUDIES OF MPFC FUNCTION}

Early studies of delayed response deficits in monkeys (Jacobsen, 1936) led investigators to focus on the role of the prefrontal cortex in working memory: the ability to hold information online and guide behavior with these internal representations (Goldman-Rakic and Selemon, 1997; Fuster, 2001). Subsequent studies identified the principal sulcus of monkey dlPFC as a critical site for visuospatial working memory (Goldman and Rosvold, 1970; Goldman et al., 1971) and ventral lateral PFC for non-spatial working memory (Goldman-Rakic, 1996; Meyer et al., 2011). Early lesion studies in the rat associated mPFC with impairments of spatial reversal, delayed response, and delayed alternation and orbitofrontal PFC with increased perseveration and responding during extinction: findings consistent with effects of dlPFC and orbitofrontal cortex lesions in non-human primates (Divac, 1971; Kolb et al., 1974; Larsen and Divac, 1978). These results led to the view that portions of rodent mPFC are homologous to primate dlPFC. Anatomical studies have challenged that view by demonstrating homology between rodent mPFC and primate anterior cingulate and premotor cortices, but not dlPFC, based on relative location, cytoarchitecture, receptor binding studies, and anatomical and functional connectivity (Preuss, 1995; Vogt et al., 2013; Schaeffer et al., 2020).

\section{Effects of mPFC Lesions on Spatial Delayed Conditional Discrimination}

Working memory is important for adaptive responding: allowing organisms to hold and manipulate information online while monitoring fluctuations in the environment (Miller et al., 2018; Cavanaugh et al., 2020). Early lesion studies indicated that $\mathrm{mPFC}$ lesions in the rat have a selective effect on working memory, impairing delayed conditional discriminations based on response-related egocentric information while sparing maze tasks that require an allocentric solution (Becker et al., 1980; Kolb et al., 1983; Kesner et al., 1996; de Bruin et al., 2001). Porter and Mair (1997) tested this distinction by comparing the effects of PFC lesions on a series of tasks trained in automated 8 arm radial mazes, beginning with an allocentric 8 arm task trained in a lighted room with many visible cues and changing in stages to end with two choice egocentric DNMTP (Figure 2). Neither mPFC nor complete PFC lesions affected the allocentric tasks, including two choice DNMTP where three arms were selected at random for each trial from the eight arms of the maze (i.e., no predictable configuration between the arms) both with 
and without visible external cues (Figures 2A,B). Both mPFC and complete PFC lesions produced stable impairment only at the last stage of training where the same three arms were used on every trial in a $\mathrm{T}$-configuration such that choice responses were defined by the egocentric direction of turning (left vs. right) from the stem of the $\mathrm{T}$ and the room was darkened and mazes covered to eliminate external allocentric visual cues (Figure 2C). Porter et al. (2000) confirmed these findings by comparing the effects of $\mathrm{mPFC}$ and hippocampal lesions on the allocentric and egocentric versions of 2-choice radial maze DNMTP. Both hippocampal and $\mathrm{mPFC}$ lesions produced delay-independent impairment for rats trained pre-surgically and tested post-surgically with the egocentric version (Figure 2D) while hippocampal lesions produced delay-dependent impairment and mPFC lesions spared the allocentric version (Figure 2E). To test possible explanations for deficits, tasks were switched after initial post-surgical testing. Interestingly, rats trained initially with the allocentric version of DNMTP were protected from effects of mPFC, but not hippocampal, lesions when switched to the egocentric version (Figures 2D,F).

These findings have several important implications. First, since egocentric and allocentric versions of radial maze DNMTP used the same deprivation procedures and water reinforcement it is unlikely that the egocentric impairment is related to reinforcement mechanisms or to the ability to utilize reinforcement-related information flexibly to select different response alternatives on different trials. Second, the ability of $\mathrm{mPFC}$ lesioned rats to perform the egocentric version following extensive allocentric training (Figure 2F) indicates that the deficits observed for egocentric DNMTP (Figures 2C,D) cannot be ascribed to the repetition of the same response alternatives on every trial. This rules out interference effects or difficulty of temporal discrimination produced by frequent repetition of response alternatives. One possibility consistent with these results is that rats were biased by pre-and post-surgical allocentric training to rely on allocentric cues or an internalized spatial map of the maze when subsequently switched to egocentric DNMTP (see Porter et al., 2000 for a discussion of this possibility).

Third, the spared ability of rats with $\mathrm{MPFC}$ or complete PFC lesions to perform allocentric DNMTP shows that the effects of mPFC lesions on egocentric DNMTP deficits cannot be ascribed to a generalized impairment of working memory. mPFC lesions in these studies involved agranular medial, anterior cingulate, and prelimbic cortices while the complete PFC lesions additionally damaged agranular insular and ventral orbital areas (cortical areas based on Öngür and Price, 2000; Heidbreder and Groenewegen, 2003). These results are consistent with reports that similar mPFC lesions impair egocentric while sparing allocentric spatial memory in other tasks (Kolb et al., 1983; Harrison and Mair, 1996; Kesner et al., 1996; de Bruin et al., 2001) and, further, spare other measures of working memory, including olfactory continuous non-matching-to-sample (Koger and Mair, 1994) and visual object memory (Kesner et al., 1996; Ennaceur et al., 1997). Ragozzino et al. (1998, 2002), report that more ventral mPFC lesions involving IL and MO areas affect working memory for allocentric spatial and visual object information. These findings are consistent with the view that different regions of PFC support different domains of working memory (Goldman-Rakic, 2005; Kesner and Churchwell, 2011). More recent evidence has shown that different modalities of working memory are mediated by other areas of the association cortex (Miller et al., 2018; Xu, 2018; Buchsbaum and D'Esposito, 2019; Cavanaugh et al., 2020) indicating that working memory may represent a property of cortex that extends beyond PFC.
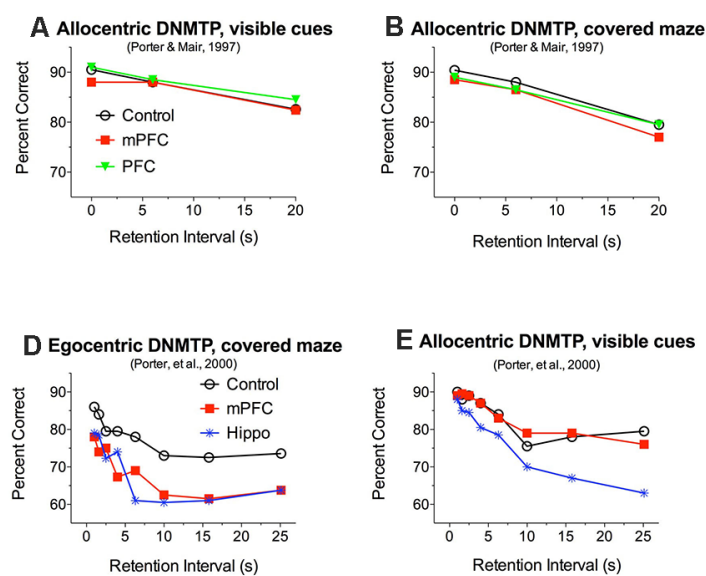
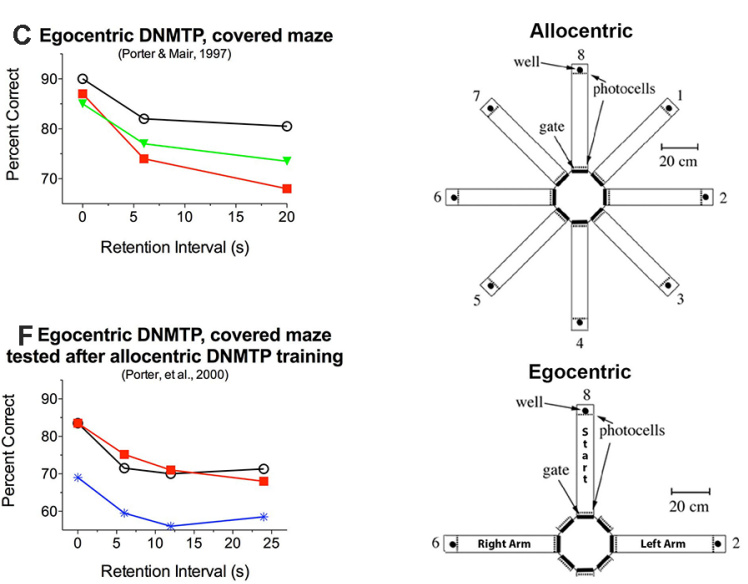

FIGURE 2 | Effects of medial prefrontal (mPFC), complete prefrontal (PFC), and hippocampal (Hippo) lesions on allocentric and egocentric versions of 2-choice DNMTP trained in eight arm radial mazes. Samples and choices in egocentric DNMTP were between the same two arms on every trial, located to the left and right of the holding arm. Arms for allocentric DNMTP were selected at random on every trial from the eight alternatives so that their configurations were unpredictable. For egocentric DNMTP external cues were minimized by covering mazes and training in the dark, while allocentric DNMTP was trained in uncovered mazes with lights on and many visible external cues. mPFC and PFC lesions impaired egocentric (C,D) and spared allocentric DNMTP (A,B,E) while hippocampal lesions impaired both tasks (D,E). Rats that received extensive allocentric training (30 presurgical and 20 postsurgical sessions) were protected when subsequently switched to egocentric DNMTP (F). Data are replotted from studies cited. 
The delay-independent effects of mPFC lesions on DMTP and DNMTP (Figures 2, 4H) are consistent with evidence that PFC lesions affect the ability to use conditional or other learned rules to select between choice alternatives independent of demands on working memory (Petrides, 1985; Winocur and Eskes, 1998; Sharpe and Killcross, 2015; Germann and Petrides, 2020). Stevens and Mair (1998) tested this idea with an auditory match-to-position (AMTP) task in which the discriminative stimulus was turned off at different times before (mnemonic) or after (non-mnemonic) the choice response. While all groups performed better for non-mnemonic conditions where stimuli were present at the time of choice, mPFC and central thalamic lesions produced comparable deficits for mnemonic and non-mnemonic choice. These results demonstrate an impairment in a trial-to-trial selection based on a learned conditional rule where the discriminative stimulus is present at the time of choice. They do not rule out a role for working memory in monitoring actions and outcomes or utilizing learned information about the conditional rule in nonmnemonic AMTP trials. Analyses of DMTP (Mair et al., 1998) and DNMTP (Harrison and Mair, 1996; Porter et al., 2000) in operant chambers that allow precise measurement of response time (RT) data have revealed significant increases in choice RT for $\mathrm{MPFC}$ lesions (Figure 5D). Other analyses in these studies showed that matching for RT did not affect deficits in response accuracy. Thus mPFC lesions appear to have two distinct effects on egocentric spatial delayed conditional discriminations: delayindependent impairment of response accuracy and slower RTs for choice responses.

\section{Effects of mPFC Lesions on Instrumental Behavior}

mPFC lesions that produce DMTP (Mair et al., 1998) or DNMTP (Harrison and Mair, 1996; Porter et al., 2000) impairments have been found to spare discrimination learning when tested subsequently for serial reversal learning where choices involved the same levers, in the same apparatus, with the same reinforcement used for the DMTP or DNMTP tasks. This suggests that mPFC lesions that affect delayed conditional discrimination spare rule-based discrimination learning where the same response alternative is associated with reinforcement on every trial. Other evidence suggests that mPFC lesions can affect rule-based responding when stimuli are difficult to discriminate (Bussey et al., 1997) or under conditions that make it difficult to detect and attend to information relevant to action selection (Birrell and Brown, 2000; Dalley et al., 2004; Chudasama, 2011; Fisher et al., 2020; Bubb et al., 2021). For instance, mPFC lesions increase RT and decrease accuracy for responding to brief luminance cues in the 5-choice serial reaction time task (Muir et al., 1996; Chudasama et al., 2003). Interestingly, more posterior (Muir et al., 1996) or ventral (Chudasama et al., 2003) mPFC lesions were found to increase premature responding in this task, indicative of impaired inhibitory control.

Similar results have been observed for a visual-spatial reaction time (VSRT) designed around the octagonal hub used for radial DNMTP tasks (Figure 3). In VSRT trials, rats entered the hub from the observation arm (see Figure 3), triggering a brief luminance cue that indicated the correct response port where a nose poke was reinforced with water. In DNMTP rats entered a similar hub from the holding arm where they were presented with open gates for two arms. They received water reinforcement for responding to the arm not reinforced on the preceding sample trial (Figure 2; see above). mPFC lesions increased RT and impaired accuracy of VSRT and produced egocentric radial maze DNMTP impairment consistent with earlier results shown in Figure 2 (Bailey and Mair, 2004). Lesions of adjacent M1 and M2 motor cortex also affected VSRT accuracy and RT but spared egocentric DNMTP. Thus, mPFC lesions affected the trial-totrial selection of spatially defined response alternatives following learned conditional rules in VSRT and egocentric DNMTP, while M1M2 lesions affected sensory-, but not a memory-guided choice. The effects of M1M2 lesions on VSRT are consistent with evidence linking motor and premotor cortices with control of intentional movements guided by external stimuli (Brown et al., 1991; Muir et al., 1996; Georgopoulos and Carpenter, 2015).

It has been argued that mPFC is important for evaluating actions and outcomes along multiple dimensions (Skvortsova et al., 2014). The anterior cingulate area of $\mathrm{mPFC}$ has been implicated in discriminating the utility of different reward options in effort-based decision-making (Walton et al., 2006; Hart et al., 2020). Consistent with this view the prelimbic area of $\mathrm{mPFC}$ has been implicated in the ability to detect actionoutcome contingencies (Balleine and O'Doherty, 2010; Balleine, 2019). Balleine and Dickinson (1998) first showed that prelimbic lesions make instrumental responding insensitive to outcome devaluation. Subsequent work found that these effects only occur with lesions made before initial training (Ostlund and Balleine, 2005; Hart and Balleine, 2016). Thus, the prelimbic cortex appears to be critical for encoding action-outcome associations but is not the location where they are stored (Balleine, 2019). More recently, Alcaraz et al. (2018) used chemogenetic methods to dissect the contributions of thalamocortical and corticothalamic projections to dorsal mPFC on encoding actionoutcome encoding. They report that thalamocortical projections are important for both outcome- and contingency-devaluation, while corticothalamic projections are important for outcome-, but not contingency-devaluation.

Fuster (2001) argued that PFC is organized to remember, plan, and execute actions: integrating perceptions and actions in time to support goal-directed behavior. Consistent with this $\mathrm{mPFC}$ lesions affect recency discriminations used to assess temporal order memory in the rat (Kesner and Churchwell, 2011). Other evidence indicates that prefrontal and premotor cortices interact with striatum to support action sequence learning in humans and non-human primates (Kennerley et al., 2004; Poldrack et al., 2005; Di Russo et al., 2017). Bailey and Mair $(2006,2007)$ showed comparable effects of frontal cortical and striatal lesions in rats trained to perform a series of nose poke responses in an action sequence learning task. For unlesioned controls repetition learning increases RT to initiate learned sequences, reflecting motor planning, and decreased RT for subsequent responses in the sequence, reflecting the benefits of habitual learning. Lesions involving agranular medial and anterior cingulate areas of $\mathrm{mPFC}$ increased $\mathrm{RT}$ to initiate learned 

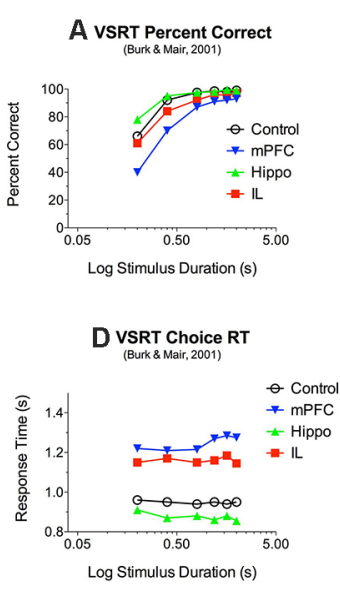
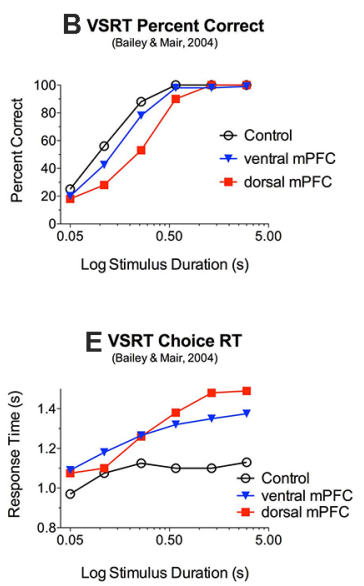
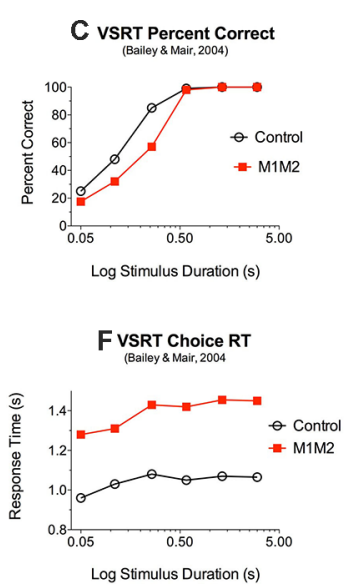

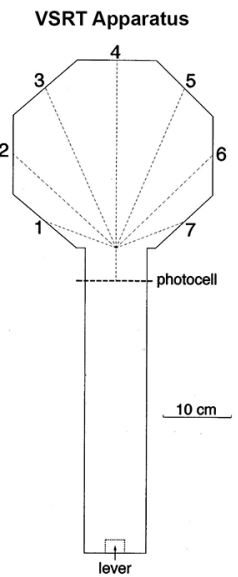

FIGURE 3 | Effects of medial prefrontal (mPFC) and motor (M1M2) cortex and rostral intralaminar (IL) thalamic lesions on reaction time (RT) and accuracy of responding to luminance cues in the visuospatial reaction time (VSRT) task. VSRT trials were initiated by a lever press at the end of the arm. A brief luminance cue was then triggered to indicate the $\mathrm{S}+$ response port when the rat crossed the photocell just before entering the octagonal hub. Water reinforcement was delivered in the S+ port if the rat entered it first within a 3.0 s limited hold. PFC and M1M2 lesions had duration-dependent effects on response accuracy (A,B,C) and increased RT for (D,E,F). IL thalamic lesions increased RT (D) but did not have a significant effect on choice accuracy (A). Data are replotted from studies cited.

sequences, suggesting a role for these areas in motor preparation, while sparing the decrease in RT for later nose pokes in learned sequences, providing evidence of spared habitual repetition learning. Parallel effects were observed for lesions in related areas of the striatum (Bailey and Mair, 2006).

Adaptive goal-directed behavior depends on multiple functions supported by mPFC. There is strong evidence for the role of $\mathrm{mPFC}$ in working memory, although it seems unlikely that this extends across all domains of information or that it is sensitive to the length of the memory delay. Beyond remembering stimulus information, working memory is important for monitoring actions and outcomes across time (see Dalley et al., 2004), holding stimulus information online to control attention, and maintaining goal-related information during motor planning. mPFC is important for flexible responding where trial-to-trial response selection is based on a learned conditional rule. mPFC has also been implicated in several attentive processes that allow animals to respond efficiently to task-relevant information. A case could also be made for a fundamental role of $\mathrm{mPFC}$ in detecting and encoding relationships between actions and their consequences and thus the capacity for adaptive goal-directed responding. Such impairment could potentially account for the profound effects of mPFC lesions on conditional responding, particularly for tasks that require flexible selection of different responses on different trials. Finally, mPFC is also thought to play an important role in organizing and executing temporal sequences of actions.

\section{BEHAVIORAL STUDIES OF CENTRAL THALAMIC FUNCTION}

mPFC interacts with multiple central thalamic nuclei to control neural networks that give rise to adaptive goal-directed behavior
(Mitchell et al., 2014; Mair et al., 2015; Halassa and Sherman, 2019; Fresno et al., 2019). To what extent do individual nuclei contribute to $\mathrm{mPFC}$ control of intentional responding? Early studies focused on $\mathrm{MD}$, in part because of prominent connections with PFC and its implication in early studies of amnesia (Victor et al., 1971; Isseroff et al., 1982; von Cramon et al., 1985; Zola-Morgan and Squire, 1985). Several lines of research broadened this focus to include contributions of other central thalamic nuclei: anatomical evidence that multiple central thalamic nuclei have afferent and efferent connections with PFC and PFC-related pathways (Groenewegen, 1988; Sesack et al., 1989; Berendse and Groenewegen, 1991; Ray and Price, 1992; Vertes, 2004; Hoover and Vertes, 2007); clinical studies associating thalamic amnesia with damage to other parts of central thalamus (Carlesimo et al., 2011; Van der Werf et al., 2003); findings from the post-thiamine deficiency (PTD) model of the Wernicke-Korsakoff syndrome that intralaminar lesions produce cognitive impairment in this model (Figures 1C,D; Mair, 1994; Mair et al., 2015); and evidence that the hippocampal-anterior thalamic axis plays a critical role in episodic memory (Aggleton and Brown, 2006). A complete review of this literature is beyond the scope of this article. Here we will focus on response-related measures of learning and memory that depend on $\mathrm{mPFC}$ in the rat.

\section{Effects of Intralaminar, Mediodorsal, and Ventromedial Nuclei on Spatial Delayed Conditional Discrimination}

Large central thalamic lesions involving intralaminar nuclei and adjacent areas of MD produce delay-independent impairment comparable to mPFC lesions, affecting both the speed and accuracy of DMTP and DNMTP choice (Mair and Lacourse, 1992; Burk and Mair, 1998). Similar deficits were observed 

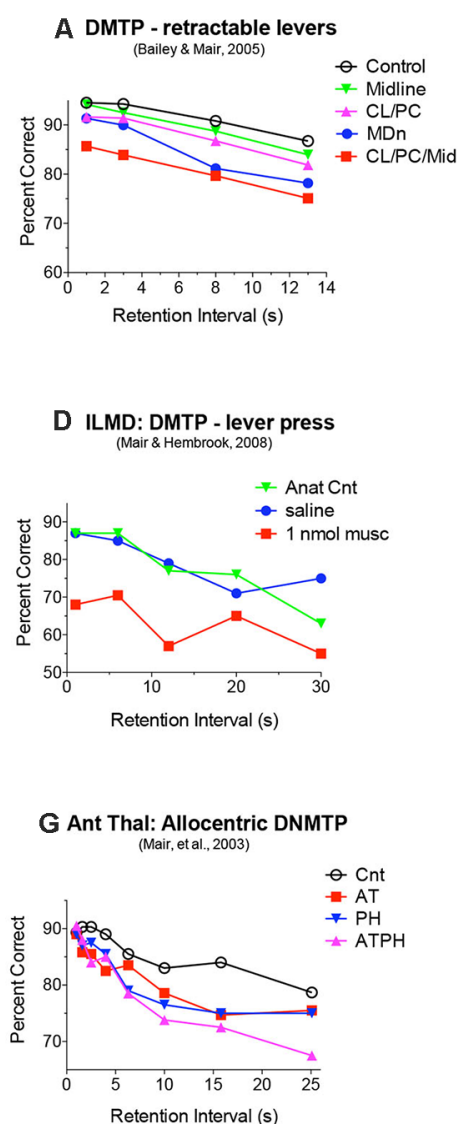
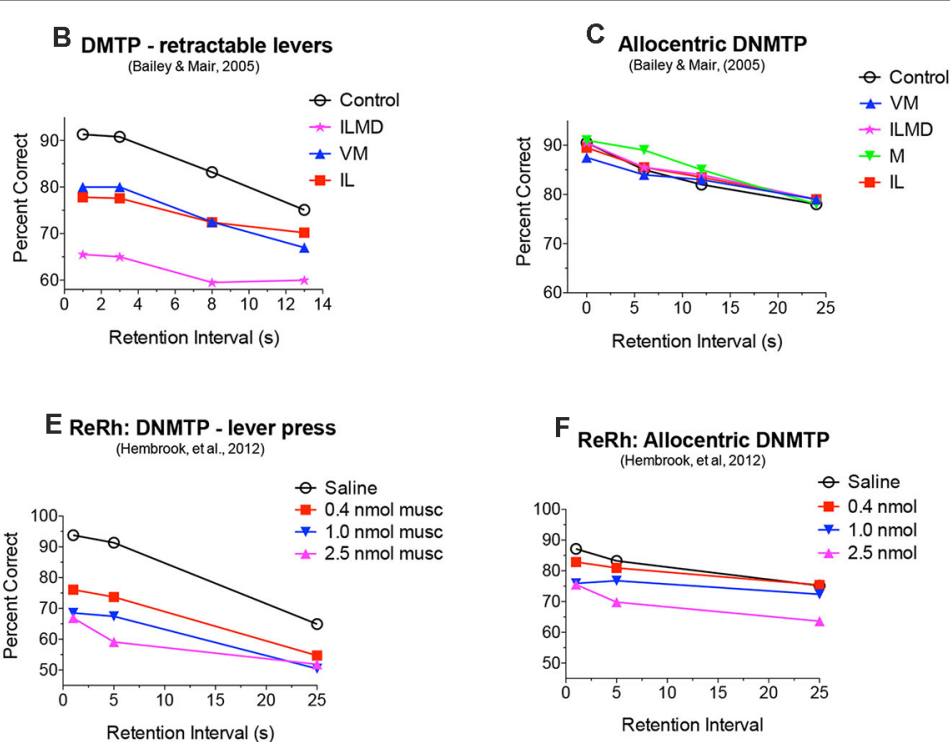

H MPFC: DMTP - retractable levers

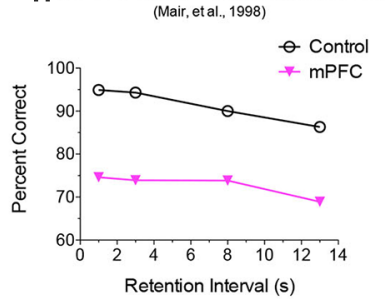

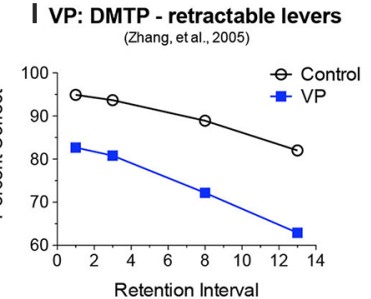

FIGURE 4 | Effects of central thalamic lesions on delayed matching (DMTP) and non-matching (DNMTP) to position accuracy. Lesions of the mediodorsal nucleus (MD) produced delay-dependent (A) and lesions of rostral intralaminar (IL), ventromedial (VM), and IL and MD combined (ILMD) produced delay-independent impairment (B) of egocentric DMTP trained with retractable levers, an impairment also observed with reversible inactivation of IL and MD (D). IL, ILMD, and VM lesions did not have significant effects on allocentric radial maze DNMTP (C). Reversible inactivation of reuniens (Re) and rhomboid (Rh) nuclei in the ventral midline thalamus produced delay-independent impairment of DNMTP trained with retractable levers at all muscimol doses tested (E) and allocentric radial maze DNMTP only at the highest dose tested (F). Anterior thalamic (AT) lesions produced delay-dependent impairment of allocentric radial maze DNMTP comparable to the effects of parahippocampal cortex (PH) lesions (G). Combined AT and PH lesions produced a larger deficit, comparable to the effects of hippocampal lesions Figure $\mathbf{2 E}$ on this task. Lesions of the medial prefrontal cortex (mPFC) and ventral pallidum (VP) produce delay-independent impairment of DMTP (H,I) comparable to the effects of IL, ILMD, and VM lesions (B). Data are replotted from studies cited.

for the PTD model (Mair et al., 1991; Robinson and Mair, 1992) where MD and intralaminar nuclei are consistent sites of thalamic pathology (Figure 1). Reversible inactivation of these nuclei with microinjected drugs can produce comparable impairment of accuracy without affecting response speed (Figure 4D; Porter et al., 2001; Mair and Hembrook, 2008). To control for the effects of response accuracy, and thus the frequency of reinforcement, on deficits produced by thalamic lesions a staircase method was used to define the memory delay producing 75\% accuracy for PTD (Robinson and Mair, 1992) and radiofrequency thalamic lesions (Mair and Lacourse, 1992). This method was successful in matching lesion and control groups for response accuracy (and thus reinforcement density) while demonstrating significant impairment in the length of retention interval producing $75 \%$ accuracy for both PTD (6.1 vs. $14.6 \mathrm{~s}$ for controls) and radiofrequency lesions (7.4 vs. $17.8 \mathrm{~s})$.
Discrete lesions targeting specific nuclei have more specific effects. Bailey and Mair (2005) compared the effects on DMTP of lesions targeting MD, intralaminar, midline, and ventromedial (VM) nuclei that were carefully positioned to avoid damaging anterior thalamic nuclei. Lesions restricted to lateral intralaminar (CL, PC) or dorsal midline nuclei did not significantly affect performance alone, but combined in the larger $\mathrm{CL} / \mathrm{PC} / \mathrm{Mid}$ lesion produced a delay independent impairment (Figure 4A) that did not affect response time (RT; Figure 5A). Lesions restricted to MD resulted in delaydependent deficits that did not affect RT. Burk and Mair (1998) found a non-significant trend towards impairment and no effect on RT for MD lesions with a similar DMTP task in which sample response requirements were manipulated. Other studies examining the effects of MD on spatial memory tasks have produced mixed results for rats and monkeys (Mitchell and Chakraborty, 2013). Young et al. (1996) found a delay-dependent 


\section{A DMTP Response Time (RT), study 1 (Bailey \& Mair, 2005)}

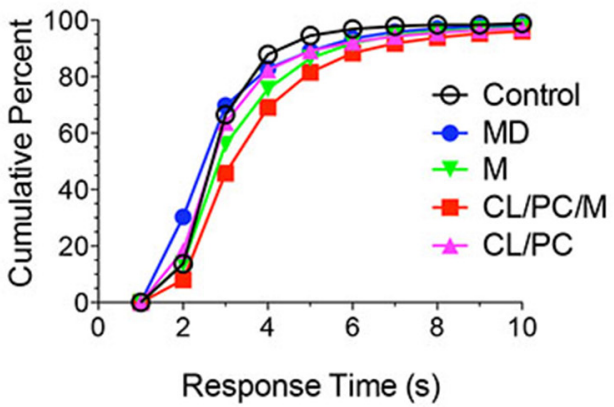

C DMTP Accuracy vs. RT, study 2 (Bailey \& Mair, 2005)

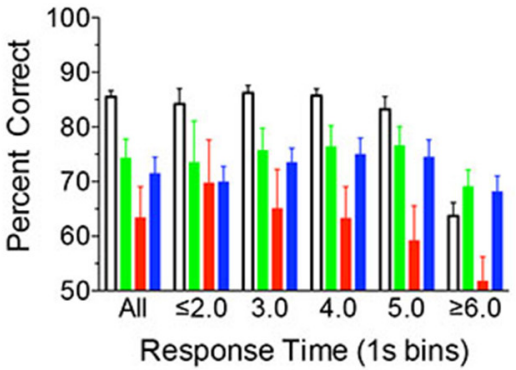

B DMTP RT, study 2

(Bailey \& Mair, 2005)

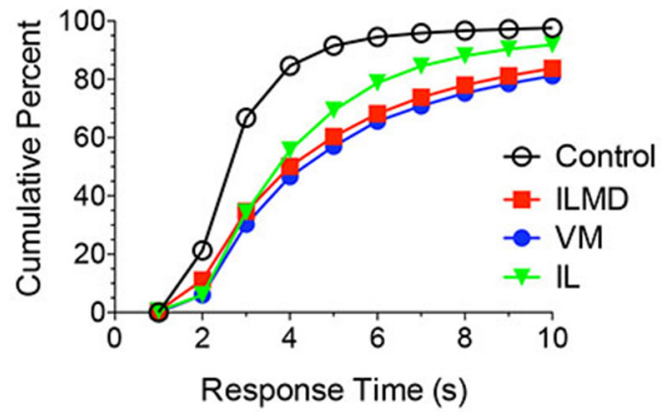

D DMTP RT : mPFC, Hippocampus (Mair, et al, 1998)

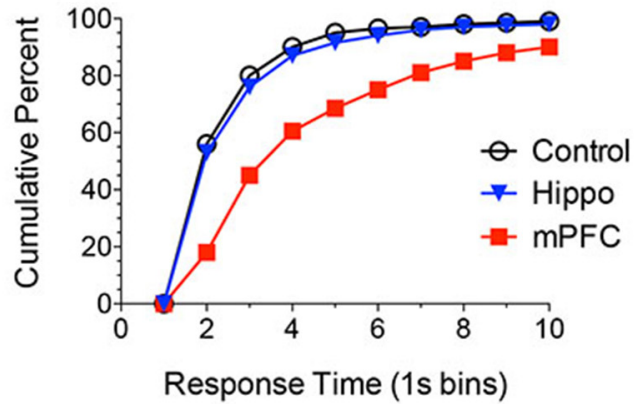

FIGURE 5 | (A,B,D) Response time (RT) analyses of DMTP choice plotted as cumulative functions. RT was measured from the lever press marking the end of the memory delay to the choice press (see Figure 6). RT was increased significantly by rostral intralaminar (IL), ventromedial (VM), and large lesions damaging IL and the mediodorsal (MD) nuclei (ILMD; B). These same lesions produced delay independent impairments of DMTP (Figure 4B). To test whether the increase in RT could account for the decrease in DMTP accuracy, separate analyses were conducted for responses divided into discrete RT bins. Restricting responses to $1 \mathrm{~s}$ time bins did not affect significant deficits produced by each of the lesions for RT bins with sufficient numbers of responses to support these analyses (C). mPFC, but not hippocampal, lesions were associated with a similar increase in DMTP RT (D) and accuracy Figure 4H. Data are replotted from studies cited.

impairment for MD lesions in a fine-grained analysis of temporal decay in an operant DNMTP task where rats were trained to stability at a series of delays and rate of decay inferred from an asymptotic performance at each delay. By contrast, Hunt and Aggleton (1998) found significant effects of MD lesions on errors to criterion learning DMTP trained in a T-maze but not for temporal decay of this task once learned. Clearly, MD lesions do not produce DMTP or DNMTP deficits comparable to the more substantial, delay-independent effects of mPFC lesions which affect accuracy and RT for these tasks (Figures 2, 4, 5).

Bailey and Mair (2005) found that complete intralaminar lesions involving CL, PC, and CM produced delay independent deficits for DMTP accuracy (Figure 4B) and RT (Figure 5B) comparable to effects of $\mathrm{mPFC}$ lesions. VM lesions also produced delay independent deficits with a more substantial increase in RT. Large ILMD lesions, involving midline, $\mathrm{MD}$, and intralaminar nuclei caused delay independent impairment about twice as severe as intralaminar (or VM) lesions (Figure 4B) and increased RT comparable to VM lesions (Figure 5B). To test whether deficits in DMTP or DNMTP accuracy are secondary to effects of thalamic lesions on response speed, separate analyses of response accuracy were conducted with restricted RT windows (Mair and Lacourse, 1992; Burk and Mair, 1998, 1999; Bailey and Mair, 2005). The results of these analyses have consistently shown effects of thalamic lesions on DMTP and DNMTP accuracy persist with RT restrictions except for long RTs where group differences are limited by floor effects (Figure 5C). Intralaminar lesions have broad effects on adaptive responding that can affect functions spared by mPFC lesions. These include olfactory continuous non-matching to sample (Koger and Mair, 1994; Zhang et al., 1998) and serial reversal learning (Mair et al., 1991; Harrison and Mair, 1996; Burk and Mair, 1998).

Bolkan et al. (2017) used optogenetic methods to demonstrate a delay-dependent effect of MD inhibition in mice for T-maze DNMTP. Interestingly they found evidence for a directional interaction where thalamocortical projections of MD support 
sustained firing in mPFC during the memory delay and corticothalamic projections the subsequent choice response. Other recent studies have shown that persistent cortical activity depends on thalamocortical loops involving $\mathrm{MD}$ and $\mathrm{mPFC}$ for attentional control (Schmitt et al., 2017) and VM for preparatory activity in the motor cortex (Guo et al., 2017). Collins et al. (2018) used optogenetics to dissect corticothalamocortical networks involving MD and VM. They report that $\mathrm{MD}$ and $\mathrm{VM}$ are excited by reciprocally-connected neurons providing layer 5 "driver" and layer 6 "modulatory" afferents from mPFC, that appear organized to activate and maintain persistent firing in thalamocortical neurons. Collins et al. also found that thalamocortical projections of $\mathrm{MD}$ strongly activate layer $2 / 3$ cortico-cortical neurons while VM provides subthreshold excitation across layers in mPFC. The predominant thalamocortical and corticothalamic connections between VM and dorsal agranular medial areas of mPFC (Vertes, 2002; Hoover and Vertes, 2007) seem consistent with the implication of VM supporting preparatory responses in the motor cortex (Guo et al., 2017). Taken together these results suggest that cortico-thalamocortical circuits support the temporary maintenance of information in $\mathrm{MPFC}$ important for adaptive responding that extends well beyond the traditional view of working memory as a temporary buffer for sensory or episodic information.

\section{Effects of Midline and Anterior Nuclei on Spatial Delayed Conditional Discrimination}

Intralaminar lesions that extend into anterior areas of the thalamus produce delay independent impairment of allocentric radial maze DNMTP (Mair et al., 1998), a task spared by intralaminar lesions that do not affect anterior thalamus (Bailey and Mair, 2005; see above). The anterior thalamic nuclei are important nodes in hippocampal-related pathways that support allocentric spatial learning and memory (Aggleton and Nelson, 2015; O’Mara and Aggleton, 2019). Multiple reports indicate that anterior thalamic lesions affect allocentric DNMTP and other measures of spatial memory spared by mPFC, intralaminar, and MD lesions (Warburton et al., 1997; Mair et al., 2003; Wolff et al., 2008). Mitchell and DalrympleAlford (2006) compared effects of anterior thalamic lesions with lateral thalamic lesions involving intralaminar and MD and found evidence of a double-dissociation consistent with these findings: anterior thalamic lesions affected the post-surgical acquisition of an allocentric spatial memory task trained in a radial maze and spared performance of a pre-surgically trained egocentric memory task, while lateral lesions of MD and the intralaminar nuclei had opposite effects. Alcaraz et al. (2016) reported an analogous double dissociation in which lesions damaging MD and intralaminar nuclei affected a spatial outcome-devaluation task while sparing an allocentric spatial memory task, while anterior nucleus lesions had the opposite effects. The severe effects of anterior thalamic lesions on spatial function have been attributed to different functions mediated by individual anterior thalamic nuclei as well as "covert effects" of anterior thalamic lesions on distributed hippocampal-related networks (Aggleton and Nelson, 2015). While AM and IAM are reciprocally connected to $\mathrm{mPFC}$, these connections are not critical for egocentric function spared by anterior thalamic lesions and insufficient to disrupt allocentric function spared by mPFC lesions.

The reuniens $(\mathrm{Re})$ and rhomboid ( $\mathrm{Rh}$ ) nuclei in the ventral midline thalamus are important sources of thalamic input to the hippocampus and MPFC that appear organized to modulate mPFC-hippocampal interactions (Vertes et al., 2006). Early evidence showed that these nuclei are important for spatial memory tasks that depend on both mPFC and hippocampus. Lesions damaging $\mathrm{Re}$ and $\mathrm{Rh}$ affect radial maze measures of spatial memory while sparing visually-guided choice in VSRT and action sequence learning (Hembrook and Mair, 2011). Localized inactivation of $\mathrm{Re}$ and $\mathrm{Rh}$ with low doses of muscimol (0.4 or $1.0 \mathrm{nmol}$ ) affects operant DNMTP, a task sensitive to the effects of both mPFC and hippocampal lesions, while sparing allocentric radial maze DNMTP, a task sensitive to the effects of hippocampal but not mPFC lesions (Hembrook et al., 2012). Subsequent studies have confirmed an important role for $\mathrm{Re}$ and $\mathrm{Rh}$ in aspects of spatial memory and cognitive control that requires the coordinated activity of the hippocampus and mPFC (Dollemanvan der Weel et al., 2019; Mathiasen et al., 2020). Both the anterior thalamic and ventral midline $\mathrm{Re}$ and $\mathrm{Rh}$ are organized to support interactions between $\mathrm{mPFC}$ and hippocampus. Anterior thalamic nuclei, with strong retrosplenial, cingulate, and mammillary body connections, appear specialized to support allocentric hippocampal function. Re and $\mathrm{Rh}$, with their extensive connections with the hippocampus and $\mathrm{mPFC}$, appear specialized to support interactions between them (Dolleman-van der Weel et al., 2019; Mathiasen et al., 2020).

\section{Effects of Thalamic Lesions on Instrumental Behavior}

Intralaminar lesions increase RT without affecting the accuracy of conditional responses to brief luminance cues in the VSRT task, while mPFC lesions increase RT and decrease accuracy (see above), and hippocampal lesions had no significant effect on RT or accuracy (Burk and Mair, 2001b). Other reports indicate that both MD (Chudasama and Muir, 2001) and Re (Prasad et al., 2013) lesions increase premature responding without affecting responses to luminance cues in the 5 choice task. This suggests a role for $\mathrm{MD}$ and $\mathrm{Re}$ on inhibitory control, but not sensory attention. The effects of intralaminar lesions on VSRT RT are consistent with evidence that intralaminar, but not $\mathrm{MD}$, lesions affect choice RT for DMTP and DNMTP tasks (Figures 5A,B; see above). The effect of MD lesions on premature responding is consistent with the effects of lesions damaging the prelimbic area of $\mathrm{mPFC}$.

Lesion studies have also demonstrated parallel effects of $\mathrm{MD}$ and prelimbic cortex lesions on action-outcome learning. Thus, MD lesions were found to abolish the effects of outcome devaluation (Corbit and Balleine, 2003). Like prelimbic lesions, MD lesions affect outcome devaluation only when made before initial training (Ostlund and Balleine, 2008). Thus, both prelimbic and MD appear to be essential for the acquisition, but 
not the expression, of goal-directed behavior. The importance of connections between the prelimbic cortex and MD for actionoutcome learning was confirmed by a crossed lesion study, where lesions damaging MD in one hemisphere, PL in the other, and contralateral projections of $\mathrm{MD}$ in the corpus callosum affected comparable to bilateral $\mathrm{MD}$ or prelimbic lesions on outcome devaluation (Bradfield et al., 2013). Recently, Alcaraz et al. (2018) used chemogenetic methods to provide evidence that thalamocortical projections from lateral MD to dorsal mPFC affect both outcomes- and contingency-devaluation, while corticothalamic pathways between these areas affect the outcome- but not contingency-devaluation.

Dorsal midline lesions damaging the PV and PT nuclei have not been associated with significant effects on egocentric DNMTP (Mair and Lacourse, 1992) or DMTP tasks (Bailey and Mair, 2005; Figures 4A, 5A). They have extensive limbic-related connections with systems that are important for instrumental behavior, including inputs from visceral-, arousal-, and emotionrelated areas in the brainstem, hypothalamus, and limbic forebrain and projections to prelimbic and infralimbic areas of mPFC, agranular insular and entorhinal cortices, subiculum, nucleus accumbens, and striatum, and the extended amygdala (Krout and Loewy, 2000a,b; Krout et al., 2001, 2002; Bayer et al., 2002; Vertes and Hoover, 2008; Kirouac, 2015). PV and PT thus appear organized to integrate information related to the behavioral state to influence brain systems that support adaptive goal-directed behavior. Functional analyses have focused more on PV than PT. These have provided evidence that PV affects reward-seeking behavior (McGinty and Otis, 2020), control of wakefulness (Ren et al., 2018), the salience of stimuli related to reward, aversion, novelty, and surprise in associative learning (Zhu et al., 2018), and conditioned and unconditioned emotional behavior (Barson et al., 2020). Lesion studies have shown that PV affects the attribution of incentive salience to reward cues in sign tracking (Haight et al., 2015).

Adaptive goal-directed responding requires organisms to plan and execute actions based on current and remembered information about the external environment, internal state conditions, and action-outcome contingencies. Central thalamic nuclei link mPFC with multiple neural networks that support these functions. No individual nucleus has proven critical for mPFC function in general. Nevertheless, lesions of specific nuclei can account for some effects of mPFC lesions, showing the importance of thalamocortical and cortico-thalamic pathways in these functions. For instance, intralaminar and VM lesions produce delay-independent impairments comparable to $\mathrm{mPFC}$, affecting RT and accuracy of egocentric DMTP and DNMTP. Lesion studies have also shown that some nuclei that are reciprocally connected with $\mathrm{mPFC}$ can affect functions spared by mPFC lesions. For instance, anterior thalamic lesions affect measures of allocentric DNMTP spared by mPFC lesions. This suggests that $\mathrm{mPFC}$ can influence functions of thalamic circuits without being a critical node in the circuit, presumably through top-down control. To elucidate functional interactions between the thalamus and cortex it is important to move beyond behavioral analyses and examine the information represented by neurons in these pathways during adaptive goal-directed behavior and how this is influenced by cortico-thalamic and thalamocortical projections.

\section{WHAT INFORMATION IS REPRESENTED BY MPFC NEURONS DURING ADAPTIVE GOAL-DIRECTED BEHAVIOR?}

Lesion studies have revealed important roles for mPFC and adjacent motor cortices in reward-guided learning and decisionmaking and provided evidence that different subregions of mPFC support specific aspects of reward-guided responding. Lesions of more dorsal areas affect memory for motor responses, response selection, and reward-guided choice while more ventral lesions of PL and IL affect allocentric and visual memory and supervisory attentional control (Dalley et al., 2004; Chudasama, 2011; Kesner and Churchwell, 2011). To what extent do mPFC neurons represent information related to functions affected by $\mathrm{mPFC}$ lesions? Are there abrupt transitions in the response properties of $\mathrm{mPFC}$ neurons that correspond with behavioral functions ascribed to dorsal and ventral subregions?

Single unit recordings in awake, behaving rats have revealed mPFC neurons with responses related to movement, actions, preparation to respond, anticipation and delivery of rewards, errors, working memory delay, and spatial location during different tasks (Jung et al., 1998; Pratt and Mizumori, 2001; Chang et al., 2002; Baeg et al., 2003; Hok et al., 2005; Euston and McNaughton, 2006; Cowen and McNaughton, 2007; Totah et al., 2009, 2013; de Saint Blanquat et al., 2010; Euston et al., 2012; Horst and Laubach, 2012; Hyman et al., 2012, 2013; Powell and Redish, 2014; Insel and Barnes, 2015). We developed the dynamic DNMTP (dDNMTP) task to examine neuronal responses related to each of these functions in a single task incorporating features known to be sensitive to mPFC lesions (Figure 6). dDNMTP is trained in open octagonal arenas with retractable levers and spouts for water reinforcement on four walls $90^{\circ}$ apart (N, E, S, W). Trials consist of a series of four lever presses. The sample phase begins with a base lever (randomly selected for each trial) extending for the start. This retracts when pressed and the sample lever extends $\left(90^{\circ}\right.$ to the left or right randomly selected). This retracts when pressed and water reinforcement is delivered from the spout immediately above. After a memory delay (randomly selected for each trial) the base lever extends again for the delay response. This retracts when pressed and the levers $90^{\circ}$ to the left and right extend for the choice response. When the lever not extended for the sample is pressed reinforcement is delivered $(*)$ and the levers retracted. The dDNMTP choice is egocentrically defined like DMTP and DNMTP tasks affected by $\mathrm{mPFC}$ lesions. The open arena provides sufficient space to characterize the movement-related neuronal activity and visible cues to examine allocentric spatial coding. By starting trials at randomly selected locations, responses related to the spatial location can be distinguished from behavioral events which shift in location for trials beginning at different base levers.

Of 1,335 isolated neurons recorded with moveable tetrode arrays, $458(34.3 \%)$ exhibited criterion event-related activity of which 445 (33.3\%) exhibited temporal patterns of activity related 


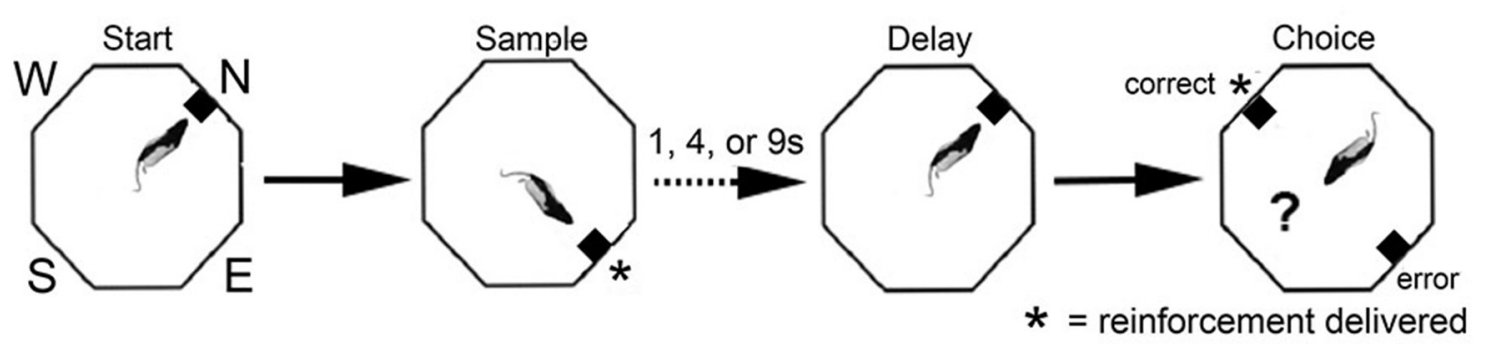

FIGURE 6 | Schematic drawing of dynamic DNMTP (dDNMTP) task. Training occurred in open octagonal chambers, equipped with levers on four walls, 90 apart, with a drinking spout above each lever to deliver reinforcement. Trials began with a randomly selected lever (N, E, S, or W) extending for the start response. The sample and correct choice levers were $90^{\circ}$ to the left or right of the start lever, which also served as the delay lever (extending at the end of the memory delay to initiate the choice response). The length of the retention interval and the direction of the sample lever ( $L$ vs. $R)$ were randomly selected for each trial. Reinforcement was given following the sample press and when the non-matching to sample lever was pressed during the choice phase. See text for details.

to actions or outcomes that were characterized as normalized population peri-event time histograms (PETH; Figures 7, 8). These included preparation to respond, movement between levers, lever-press responses, reinforcement anticipation, reinforcement delivery, errors, and memory delays. Actionrelated responses (Figure 7) included 129 that fired during periods of movement, 58 that fired during lever press responses, and 44 that fired during preparation before the start response. Movement-related responses included neurons that fired during all periods of movement between levers (M1; $n=97)$ and others that were directionally specific and fired during movements from the base to the sample and from the base to the choice levers (M2; $n=32$ ). Lever press-related responses included neurons firing during all four lever presses (LPE; $n=28)$ and others that fired only during base lever presses (BLP; $n=30$ ).

Outcome related responses $(N=191)$ included reinforcement anticipation (RA; $n=50$ ) that fired beginning $0.7 \mathrm{~s}$ before predictable times of reward and persisted for an average of $2.7 \mathrm{~s}$ throughout reward delivery; reinforcement excitation (RE, $n=63$ ) that fired within $0.2 \mathrm{~s}$ after reward delivery and remained elevated for an average of $3.0 \mathrm{~s}$; error responses $(\mathrm{E} ; n=4)$ that fired within $0.2 \mathrm{~s}$ of when the expected reward was not delivered; delay (D) responses $(n=58)$ that started firing within $0.4 \mathrm{~s}$ of when sample rewards were delivered and continued until the delay lever press; and post-reinforcement (PR) responses $(n=16)$ that began after reward delivery ended when rats disengaged from drinking spouts where rewards were delivered.

Spatial mapping of neuronal activity during dDNMTP revealed areas of activation consistent with event-related analyses, thus neurons firing during lever presses or reinforcement have higher activity in locations of response levers, and reward spouts and movement-related responses are associated with elevated activity on pathways between levers. Some neurons fire in all possible locations where associated events occur while others are spatially-restricted, firing in a subset of possible locations. Figure 9 (from Onos et al., 2016) shows examples of spatially-restricted responses. Spatial heat maps and event-related rasters and PETHs are shown for three neurons with delay related responses $(\mathbf{A}, \mathbf{G} ; \mathbf{B}, \mathbf{H}$; and F,L) along with single examples for base lever press (C,I), reinforcement excitation $(\mathbf{D}, \mathbf{J})$, and reinforcement anticipation $(\mathbf{E}, \mathbf{J})$. Although the data were insufficient for vector or decoding analyses (Georgopoulos and Carpenter, 2015; Yin et al., 2018) these results are consistent with a population code representing information about actions and their location in allocentric space.

Histological analyses show substantial overlap between the distributions of all response types in mPFC. Statistical analyses revealed biases between dorsal and ventral mPFC, with dorsal areas having more neurons with motor-related responses, including movement between all levers (M1), lever presses (both LPE and BLP), and preparatory responses (Figure 10). Ventral areas had relatively high concentrations of neurons firing during movement towards rewards (M2), delay periods following reinforcement, reinforcement anticipation (RA), and post-reinforcement (PR). There was a relatively even distribution of neurons firing during reinforcement delivery (RE) consisting of about $15 \%$ of event-related responses in dorsal and ventral compartments (Francoeur and Mair, 2018). Thus, while there are differences in the broad distributions of different response types between dorsal and ventral mPFC, there was no evidence of an abrupt transition in the types of information represented in different regions of $\mathrm{mPFC}$.

Each of the response types observed in rodent mPFC during dDNMTP represents task-specific aspects of goal-directed behavior that are consistent with $\mathrm{mPFC}$ functions identified by behavioral analyses of lesion effects. PFC relies on working memory to temporarily maintain information not available to the senses to support adaptive goal-directed responding. This is thought to be represented by persistent neuronal firing during delay intervals (Fuster and Alexander, 1971; Fuster, 2001; Goldman-Rakic, 2005) although evidence has been presented that working memory may also be implemented by sequential neuronal activation (Rajan et al., 2016). Working memory for cognitive information is associated with delayrelated activity in primate dlPFC (Fuster and Alexander, 1971; Fuster, 2001; Goldman-Rakic, 2005). Enel et al. (2020) have recently presented evidence that delay-related activity in monkey anterior cingulate cortex, the likely homolog of rodent mPFC (Preuss, 1995; Vogt et al., 2013; Schaeffer et al., 2020), represents information about reward value. This is consistent with the 

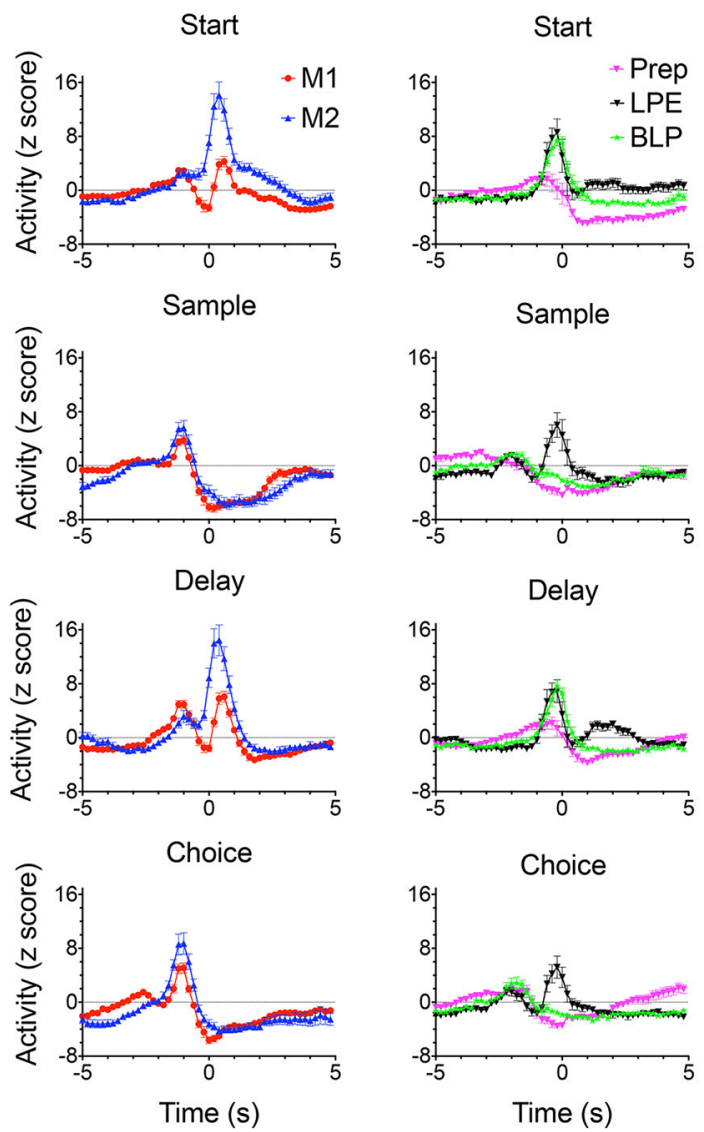

FIGURE 7 | Normalized population PETHs based on all examples observed of action-related responses. Movement 1 (M1) fired during movements toward each lever in the sequence $(N=97)$. Movement $2(\mathrm{M} 2)$ fired during movements toward the sample and choice levers and were directionally specific $(N=32)$. Lever press excitation (LPE) fired during each of the 4 lever presses in the sequence $(N=28)$. Base lever press $(B L P)$ fired during start and delay lever presses $(N=30)$. Preparatory (Prep) exhibited activity that ramped up to a peak just before the start and (to a lesser extent) the delayed responses $(N=44)$. Activity is aligned with each of the lever presses and plotted for $5 \mathrm{~s}$ before until $5 \mathrm{~s}$ after the aligned event. Error bars represent the standard error of the mean. Data are replotted from Francoeur and Mair (2018).

finding of delay period activity related to reinforcement delivery in $\mathrm{mPFC}$ during the dDNMTP task (Figure 8). At least three other response types represent information not immediately available to the senses presumably held in working memory. These include preparatory responses before the start response, reinforcement anticipation before sample and choice responses, and post-reinforcement responses.

The preponderance of neurons with responses related to actions and outcomes is consistent with evidence implicating mPFC in action-outcome contingency: clearly, mPFC neurons represent information required for this function. Similarly, the concentration of neurons with preparatory responses in dorsal mPFC for dDNMTP (Figure 7) and other tasks (Jung et al., 1998; Chang et al., 2002; Totah et al., 2009, 2013) is in keeping with evidence that lesions here selectively affect the
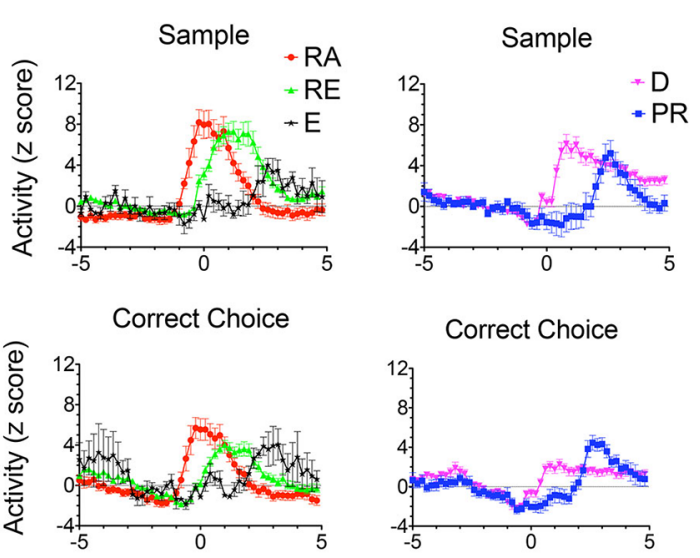

Correct Choice
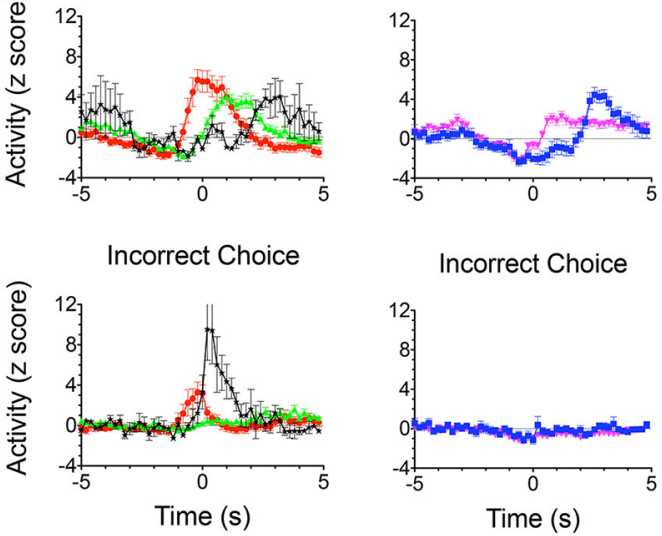

Incorrect Choice

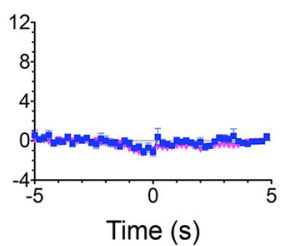

FIGURE 8 | Normalized population PETHs based on all examples observed of outcome-related responses. Each of these responses differentiated reinforced sample and correct choice responses from unreinforced incorrect choices. Reinforcement anticipation (RA) responses began 0.6-0.8 s before sample and choice responses and lasted until $1.6 \mathrm{~s}$ after reinforcement began or $0.2 \mathrm{~s}$ after errors when reinforcement was not delivered $(N=50)$. Reinforcement excitation (RE) began when reinforcement was delivered and lasted an average of $2.7 \mathrm{~s}(N=63)$. Error $(E)$ responses lasted for an average of $1.6 \mathrm{~s}$ after incorrect responses when expected reinforcement was not delivered $(N=4)$. Delay-related responses exhibited increased activity lasting across the memory delay, on average from 0.4 to $5.0 \mathrm{~s}$ after sample reinforcement and 0.2-1.6 s following correct choice reinforcement $(N=58)$. Some delay-related had spatially-restricted firing patterns (Figures 9A,B,F) and thus potentially carried information about the location of sample reinforcement across the delay interval sufficient to support a correct DNMTP choice. Post-reinforcement responses lasted on average from 2.3 to $3.6 \mathrm{~s}$ after reinforcement began, a time when rats tended to disengage after consuming reinforcement from the drinking spout $(N=16)$. Activity is aligned with each of the lever presses and plotted for $5 \mathrm{~s}$ before and after the aligned event. Error bars represent the standard error of the mean. Data are replotted from Francoeur and Mair (2018).

initiation of learned action sequences (Bailey and Mair, 2007). The importance of mPFC for organizing temporal sequences of behavior is supported by normalized population PETHs (Figures 7,8 ) that reveal a cascade of tightly coupled neuronal responses that effectively tile the temporal interval between initial preparation to when rats disengage from reinforcement following choice responses. Finally, recording studies have consistently shown large populations of neurons tuned to respond to task-relevant information. Here 445/1,335 isolated neurons exhibited one of ten discrete response types related specifically to the arbitrary actions and outcomes of dDNMTP (Figures 7, 8). Similar numbers are reported for the proportion of mPFC neurons exhibiting task-specific event-related activity for different behavioral tasks (Jung et al., 1998; Pratt and Mizumori, 


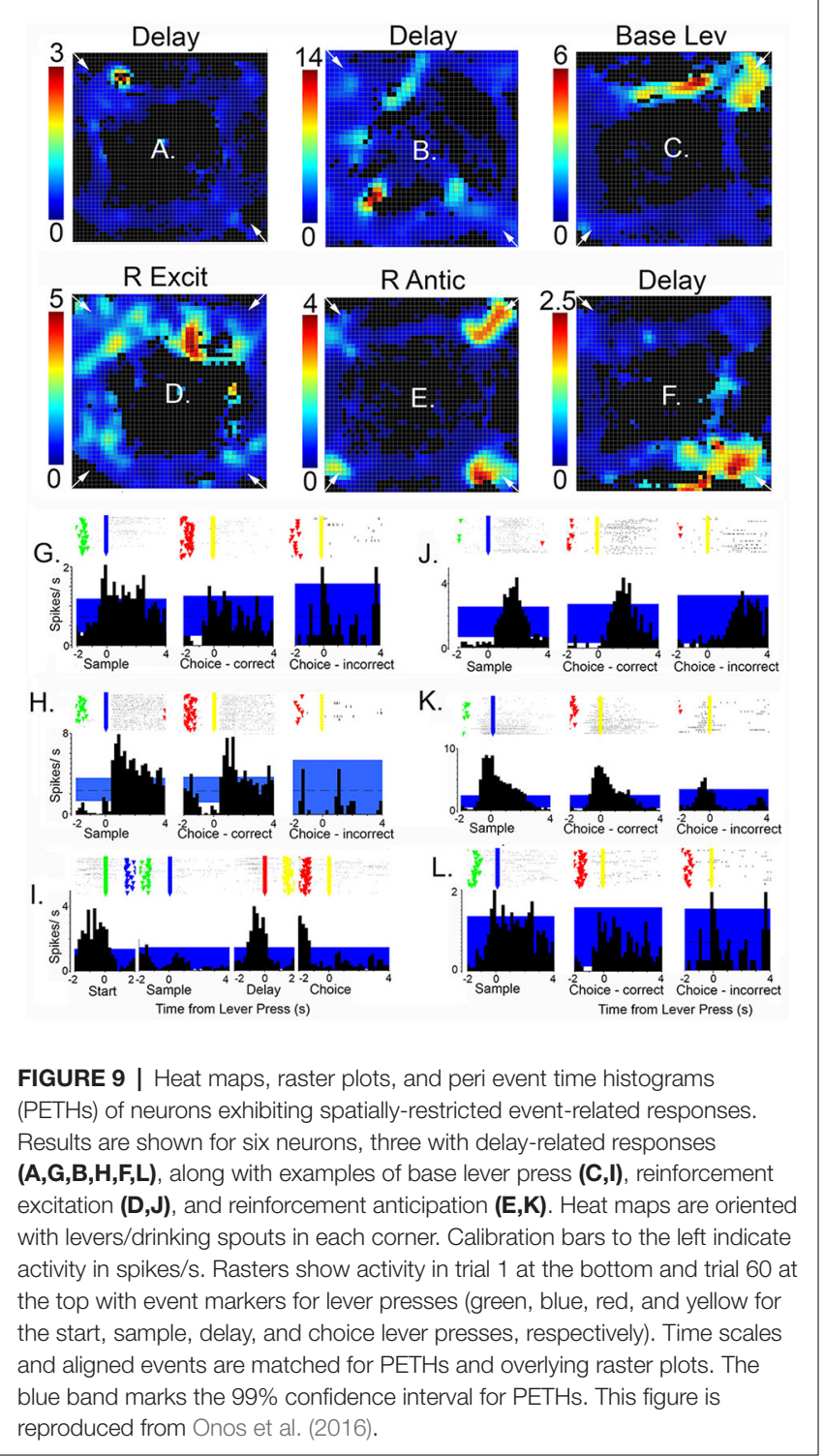

2001; Chang et al., 2002; Baeg et al., 2003; Hok et al., 2005; Euston and McNaughton, 2006; Cowen and McNaughton, 2007; Totah et al., 2009, 2013; de Saint Blanquat et al., 2010; Euston et al., 2012; Horst and Laubach, 2012; Hyman et al., 2012, 2013; Powell and Redish, 2014; Insel and Barnes, 2015). These tuning properties of $\mathrm{mPFC}$ neurons seem consistent with evidence that $\mathrm{mPFC}$ lesions affect the ability of rats to detect and discriminate information relevant to adaptive action selection (Birrell and Brown, 2000; Dalley et al., 2004; Chudasama, 2011; Fisher et al., 2020; Bubb et al., 2021).

Electrophysiological analyses of neuronal activity in awake, behaving animals support the homology of rodent mPFC with primate anterior cingulate and premotor cortices. Premotor neurons in monkeys have been shown to encode movementrelated information in extrinsic coordinates related to actions rather than muscle-like activity (Kakei et al., 2001, 2003), with imprecise coding of directional or spatial information in

\section{Distribution of Response Types}

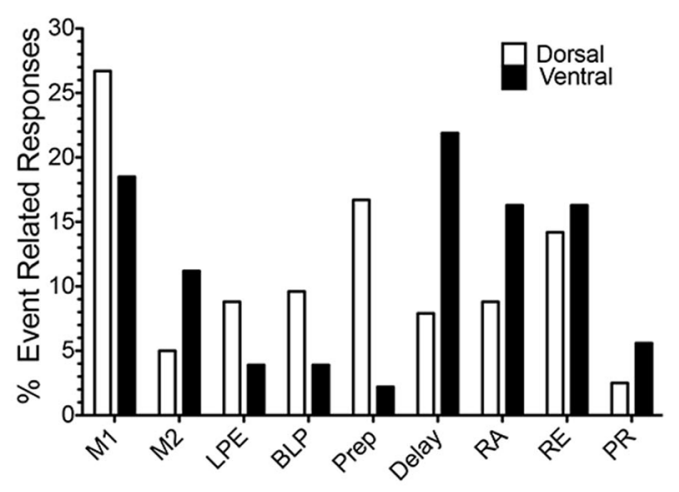

Standard Residuals

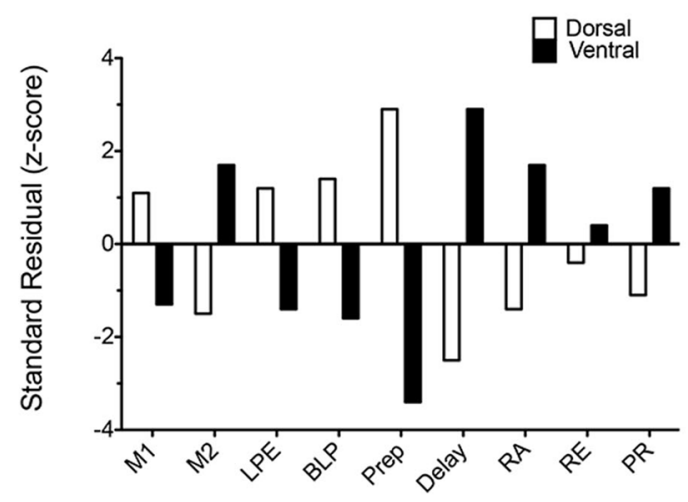

Response Type

FIGURE 10 | Distribution of response types observed in dorsal and ventral areas of mPFC. The upper plot compares the percent of responses in each compartment exhibiting each of the most common response types observed during dDNMTP. The lower plot shows standard residuals indicating the contributions of each response type to the significant chi-square comparing the distribution of different response types in dorsal and ventral mPFC. Abbreviations are the same as for Figure 11. Figure is reproduced from Francoeur and Mair (2018).

single neurons that presumably rely on population coding to achieve precision (Georgopoulos and Carpenter, 2015; Yin et al., 2018). Motor responses are preceded by preparatory activity related to motor planning (Shenoy et al., 2013; Murakami and Mainen, 2015). Other reports have described neuronal responses that precede expected reinforcement or mark the delivery or absence of expected reinforcement in monkey motor, premotor, and anterior cingulate cortices (Roesch and Olson, 2003; Amiez et al., 2006; Matsumoto et al., 2007; Marsh et al., 2015) as well as human medial PFC (Domenech et al., 2020). Recently, Enel et al. (2020) reported that delay-related activity in monkey anterior cingulate represents information about the expected value of action outcomes. This homology is also consistent with recent results from resting-state fMRI analyses that rodent $\mathrm{mPFC}$ has stronger connections with motor areas of cortex than the more broadly distributed 


\section{Distribution of Response Types}

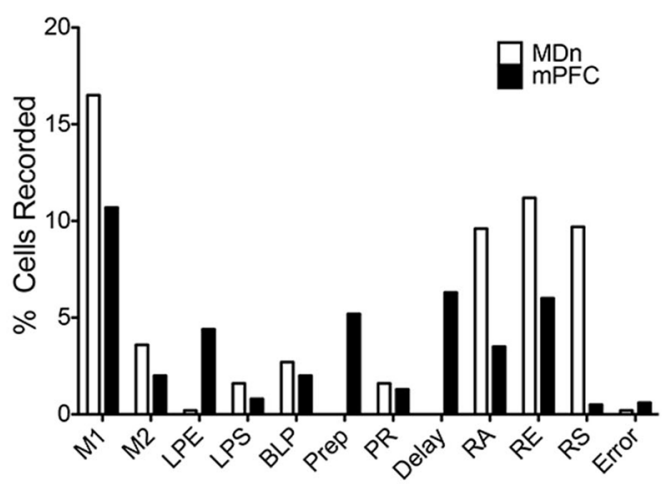

Standard Residuals

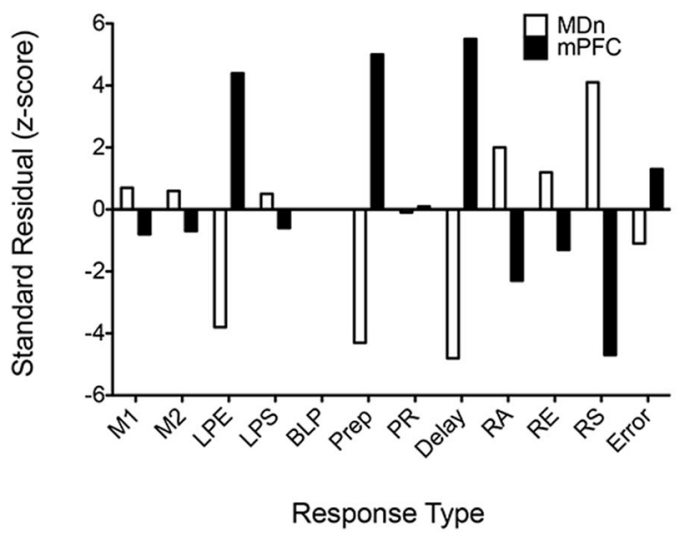

FIGURE 11 | Distribution of response types observed in the mediodorsal thalamic nucleus (MDn) and mPFC during the dDNMTP task. Standard residuals indicate the contributions of each response type to the significant chi-square comparing the distribution of different response types in MDn and mPFC. Results are shown for movement 1 (M1) and 2 (M2), lever press excitation (LPE) and suppression (LPS), base lever press (BLP), preparation (Prep), post-reinforcement (PR), error (E), and reinforcement anticipation (RA), excitation (RE), and suppression (RS). The figure is reproduced from Miller et al. (2017).

connections of primate mPFC: an organization in the rat more closely related to premotor than dIPFC areas of primates (Schaeffer et al., 2020).

\section{HOW DO CENTRAL THALAMIC NUCLEI INFLUENCE EVENT-RELATED RESPONSES OF MPFC NEURONS?}

Cortical projections excite the thalamus through driver and modulatory projections from layers 5 and 6 of mPFC. Thalamic projections activate excitatory cortico-cortical neurons and inhibitory interneurons to enhance cortical connectivity and thus regulate the activity of mPFC neurons (Cruikshank et al., 2012; Rovó et al., 2012; Bolkan et al., 2017; Schmitt et al., 2017; Collins et al., 2018; Huo et al., 2020; Lee et al., 2020). To what extent do central thalamic neurons exhibit patterns of behavioral eventrelated activity comparable to $\mathrm{mPFC}$ ? How does the activity of central thalamic nuclei affect action- and outcome-related responses of $\mathrm{mPFC}$ neurons?

Early studies revealed similar patterns of elevated firing during memory delays for neurons in MD and dlPFC in monkeys performing delayed response tasks: activity hypothesized to represent information held on-line in working memory (Fuster and Alexander, 1971; Tanibuchi and Goldman-Rakic, 2003; Watanabe and Funahashi, 2004a,b). Delay-related activity in primate MD differs from dlPFC in representing information about motor responses rather than sensory cues. Population vector analyses indicate that MD responses shift from sensoryto motor-related responses during the delay interval, suggesting a role for $\mathrm{MD}$ in constructing prospective memory information in dlPFC (Watanabe and Funahashi, 2012). Neurons in adjacent areas of oculomotor thalamus, including the rostral intralaminar nuclei, exhibit visual- and motor saccade-related responses during memory-guided saccade or anti-saccade tasks that resemble responses in reciprocally-connected areas of frontal eye fields in several important ways (Wyder et al., 2003; Tanibuchi and Goldman-Rakic, 2005; Tanaka and Kunimatsu, 2011). Neurons in the oculomotor thalamus differ from frontal eye fields in exhibiting responses more strongly related to movement information and in their sensitivity to differences in cognitive or behavioral demands (Costello et al., 2016). Studies of primate MD and oculomotor thalamus have focused primarily on sensory- and motor-related responses observed in dIPFC and frontal eye fields and not on reward-related responses observed in reciprocally-connected areas of $\mathrm{mPFC}$ (Amiez et al., 2006; Matsumoto et al., 2007; Enel et al., 2020).

The limited evidence available for the rat, suggests that MD neurons represent task-relevant sensory, motor, and reinforcement information (Oyoshi et al., 1996; Han et al., 2013; Courtiol and Wilson, 2016). Miller et al. (2017) compared neuronal responses in $\mathrm{MD}$ directly with earlier results for $\mathrm{mPFC}$ for rats performing the dDNMTP task. Of 1179 isolated neurons in nine rats, 254 (22\%) exhibited criterion event-related responses, $237(20.1 \%)$ with temporal patterns that matched response types in $\mathrm{mPFC}$ (Figures 7,8 ). The percentage of corresponding responses is consistent with the strong excitatory projections of $\mathrm{mPFC}$ to $\mathrm{MD}$. There were disparities in the relative number of different response types (Figure 11): MD had more responses related to movement ( $45 \%$ vs. $29 \%$ for $\mathrm{mPFC}$ ) and reinforcement (51\% vs. $27 \%$ ), relatively few related to lever press actions (2.1\% vs. $14.9 \%$ ), and no responses spanning the memory delay (vs. $12.7 \%$ for PFC) or during preparation before the start response (vs. 9.6\% for mPFC). The lack of preparatory activity in MD may reflect the role of $\mathrm{VM}$ as a thalamic hub for circuits supporting motor preparation (Guo et al., 2017). Choice in dDNMTP is defined by movements towards levers (Figure 6). Choice responses in dDNMTP are associated with increased frequency of movement-related responses and decreased frequency of lever press responses (Francoeur and Mair, 2020). Thus, the preponderance of MD responses related to movement and reward in dDNMTP is 
indicative of a role in the reward-guided choice. These results are consistent with evidence from studies of monkeys that MD contains numerous neurons that represent information about forthcoming movements during choice responses in ocular delayed response tasks (Watanabe and Funahashi, 2012). The large proportion of MD neurons with reward-related responses during dDNMTP is in keeping with the prominence of rewardrelated responses in mPFC (Figures 8, 9) and convergent inputs to MD from reward-related areas in the orbitofrontal cortex, ventral pallidum, and amygdala. The lack of delayrelated responses in $\mathrm{MD}$ is surprising given the prominence of these responses in primate MD during delayed response tasks. This may reflect distinct properties of primate dlPFC (Watanabe and Funahashi, 2012), that lack a homolog in the rodent brain.

Analyses of normalized population histograms reveal close correspondence in temporal patterns of activity in MD and MPFC. Figure 12 shows normalized PETHs for the two response types observed most frequently in both $\mathrm{MD}$ and mPFC during dDNMTP: movement between all lever presses (M1) and reinforcement excitation (RE). The close timing of these responses seems consistent with the strong excitatory projections from mPFC to MD and the reciprocal thalamocortical projections from MD to mPFC. Reinforcement suppression responses stand out as the one conspicuous MD response type that does not have a corresponding population in mPFC (Figure 11). Ventral pallidum (VP) contains neurons that fire in response to rewards and their predictive stimuli (Ahrens et al., 2016; Ottenheimer et al., 2018; Richard et al., 2018) and provides a robust inhibitory projection to MD that could potentially contribute to these responses (Root et al., 2015). We have recently recorded neuronal activity of VP neurons in rats performing the dDNMTP task and found that $117 / 177$ (68\%) of neurons with criterion event-related responses exhibit elevated firing when the reward is delivered, consistent with the timing of reinforcement suppression responses in MD (Krell, 2020).

Francoeur et al. (2019) examined the effects of central thalamic inactivation on mPFC by injecting muscimol at sites (affecting MD and IL) and doses previously found to produce delay independent impairment for DMTP (Figure 4D; Mair and Hembrook, 2008) and sensory-guided choice for VSRT (Newman and Mair, 2007) when applied bilaterally. To avoid disrupting behavior, which is necessary to characterize dDNMTP event-related responses, we inhibited the central thalamus unilaterally and recorded the activity of mPFC neurons in the ipsilateral mPFC. The effects of thalamic inhibition were examined by comparing the activity of single neurons across three sessions, 1 day apart: baseline (no injection), thalamic inhibition (unilateral muscimol injection), and recovery (no injection). Central thalamic inhibition increased the average firing rate for some mPFC neurons and reduced it for others while broadly suppressing event-related responses for actions and outcomes. Figure 13 (from Francoeur et al., 2019) shows results for an mPFC neuron with a reinforcement anticipation response that exhibited increased activity with thalamic inhibition. Figures 13A-C show waveforms recorded at each microwire electrode for all action potentials in each $60 \mathrm{~m}$ recording session, with the 3D cluster plots, and inter-spike interval (ISI) histograms recorded. These confirm the identity of the neuron recorded across the 3 days and show the decrease in ISI as activity increased during day 2 inactivation for this neuron. Panels $\mathrm{D}$ to $\mathrm{L}$ in Figure 13 show raster plots and normalized PETHs on day 1 (D,G,J), day $2(\mathbf{E}, \mathbf{H}, \mathbf{K})$, and day 3 (F,I,L) aligned with reinforced sample $(\mathbf{D}, \mathbf{E}, \mathbf{F})$ and correct choice $(\mathbf{G}, \mathbf{H}, \mathbf{I})$ and unreinforced incorrect choice $(\mathrm{J}, \mathrm{K}, \mathrm{L})$. Event-related responses observed on day 1 largely disappeared with thalamic inhibition on day 2 and recovered substantially on day 3 . Averaged across all neurons studied, day 2 thalamic inhibition reduced normalized activity during critical response windows to $46.9 \%$ of the day 1 response and this recovered to an average of $79.5 \%$ during day 3 recovery. Mixed model ANOVAs revealed significant effects of inactivation on day 2 and significant recovery on day 3 based on normalized activity during critical response windows. These effects did not interact with response type, the effect of thalamic inhibition on average firing rate (increased, decreased, or unchanged), location of neuron in dorsal vs. ventral mPFC, or muscimol dose. These results show that dDNMTP event-related responses are reduced nonspecifically in $\mathrm{mPFC}$ with behaviorally-significant inactivation of MD and IL.

Optogenetic studies have provided evidence that MD amplifies and sustains behaviorally-relevant information in PFC (Bolkan et al., 2017; Schmitt et al., 2017; Parnaudeau et al., 2018). These results suggest that MD may help tune $\mathrm{mPFC}$ neurons to respond to task-relevant information during adaptive goal-directed behavior. To test this possibility, we compared the effects of unilateral MD lesions made before and after initial dDNMTP training. Neuronal responses were then compared in ipsilateral (experimental) and contralateral (control) mPFC (Francoeur, 2019). The unilateral lesions did not have significant effects on behavioral performance. MD lesions made before training were associated with decreased activity of all mPFC neurons in the lesioned hemisphere and a shift in the predicted direction for event-related responses: namely more lever-press-related and fewer movement-related responses in the lesioned hemisphere. MD lesions made after initial training affected the activity of neurons with criterion event-related responses, but not neurons with uncorrelated activity. Lesions made after initial training did not affect the distribution of response types in $\mathrm{MPFC}$ in the lesioned vs. unlesioned hemisphere.

The available results suggest that the central thalamus has important short-term and long-term effects on mPFC function during adaptive goal-directed behavior. In the short term, MD amplifies and sustains neuronal responses in $\mathrm{mPFC}$ representing task-relevant information (Bolkan et al., 2017; Schmitt et al., 2017; Parnaudeau et al., 2018). Consistent with this, MD lesions produce delay-dependent impairment of response-related DMTP and DNMTP and other tasks that require flexible responses when action-outcome contingencies change. MD lesions made after initial dDNMTP training affect the activity of mPFC neurons with criterion event-related 

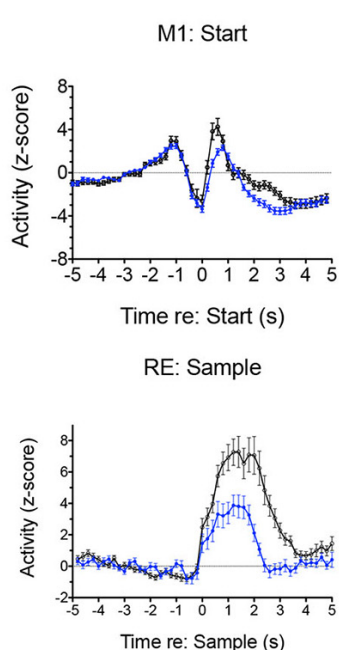

M1: Sample

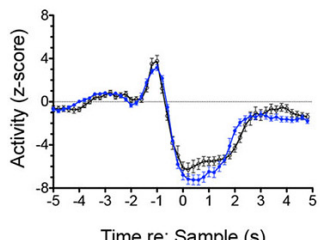

Time re: Sample (s)
M1: Delay

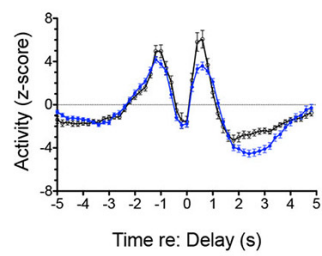

Correct Choice

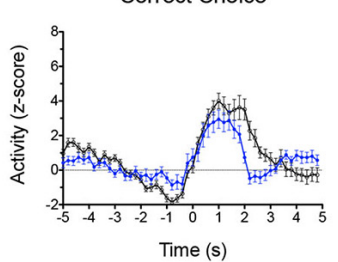

M1: Choice

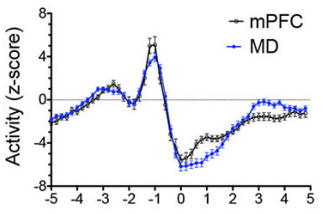

Time re: Choice (s)

Incorrect Choice

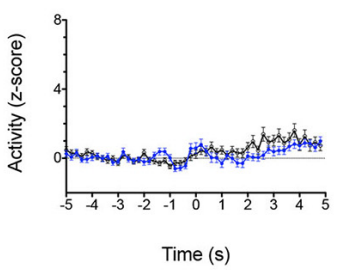

FIGURE 12 | Normalized population PETHs comparing the two most common responses in mediodorsal thalamus (MD) and medial prefrontal cortex (mPFC). Movement 1 (M1) responses aligned with the start, sample, delay, and choice lever presses are based on all samples observed in MD $(n=91)$ and $\mathrm{mPFC}(n=97)$. Reinforcement excitation (RE) responses aligned with reinforced sample and correct choice and unreinforced incorrect choice responses are based on all examples observed in MD ( $n=47)$ and mPFC ( $n=63)$. Error bars represent the standard error of the mean. Data are replotted from Miller et al. (2017).

responses, an effect that could potentially contribute to these behavioral deficits. MD lesions made before initial dDNMTP are associated with fewer movement-related and more lever pressrelated responses in $\mathrm{mPFC}$ of the lesioned than the unlesioned hemisphere. This provides evidence of a longer-term impact of $\mathrm{MD}$ on $\mathrm{mPFC}$ function and suggests that one function of $\mathrm{MD}$ is to tune $\mathrm{mPFC}$ neurons to respond to task-specific information important for adaptive responding. This finding seems consistent with evidence that MD lesions made before (but not after) initial training affects the sensitivity of rats to outcome-devaluation, a hallmark of goal-directed action (Balleine, 2019).

Adjacent IL nuclei have more widespread projections than $\mathrm{MD}$, targeting layer 1 of $\mathrm{mPFC}$ and related areas of the cerebral cortex and providing the main thalamic input to the striatum. Lesions or inactivation of the IL nuclei, which inevitably affect juxtaposed areas of MD, produce delay independent impairments of DNMTP and DMPT, increase RT for sensoryguided responding in the VSRT task, and can interfere with habitual, rule-based learning. Inactivation of IL and MD has variable effects on $\mathrm{mPFC}$ activity, increasing firing of some neurons and reducing firing of others, and has broad effects on the expression of diverse dDNMTP-related responses in mPFC. These results are consistent with the hypothesis that these nuclei regulate information transmission in cortico-cortical and cortico-basal ganglia circuits that give rise to goal-directed behavior (Saalmann, 2014; Perrin and Venance, 2019).

\section{CONCLUSIONS}

1. Medial prefrontal cortex (mPFC) supports multiple functions required for adaptive goal-directed behavior: working memory, flexible trial-by-trial response selection, attending to task-relevant information, encoding relationships between actions and their consequences, and organizing and executing action sequences. mPFC lesions produce delay-independent impairments of egocentric (response-related) DMTP and DNMTP tasks that affect RT and accuracy of responding. They spare comparable allocentric tasks.

2. During the dDNMTP task, mPFC functions are served by discrete populations of neurons with responses related to preparation to respond, movements between levers, lever press responses, reinforcement anticipation, delivery of or lack of expected reinforcement, and memory delay following reinforcement. Population analyses show that these different response types effectively tile the temporal interval from when dDNMTP trials are initiated until they end.

3. No individual thalamic nucleus can fully account for the broad effects of mPFC lesions on adaptive goal-directed behavior. Lesions of specific nuclei have distinct effects on behavior consistent with their anatomical connections.

(a) MD has very limited effects on egocentric DMTP or DNMTP tasks that depend on mPFC. While some reports find no significant effect of MD lesions on these tasks, others have described delay-dependent deficits that spare RT or impaired acquisition that disappears with training. The reports of delaydependent deficits are consistent with evidence that MD sustains and amplifies neuronal responses that represent behaviorally-relevant information in mPFC. Impairments in the acquisition are consistent with evidence that MD interacts with PFC to detect and encode action-outcome contingencies that are the basis of goal-directed learning. 

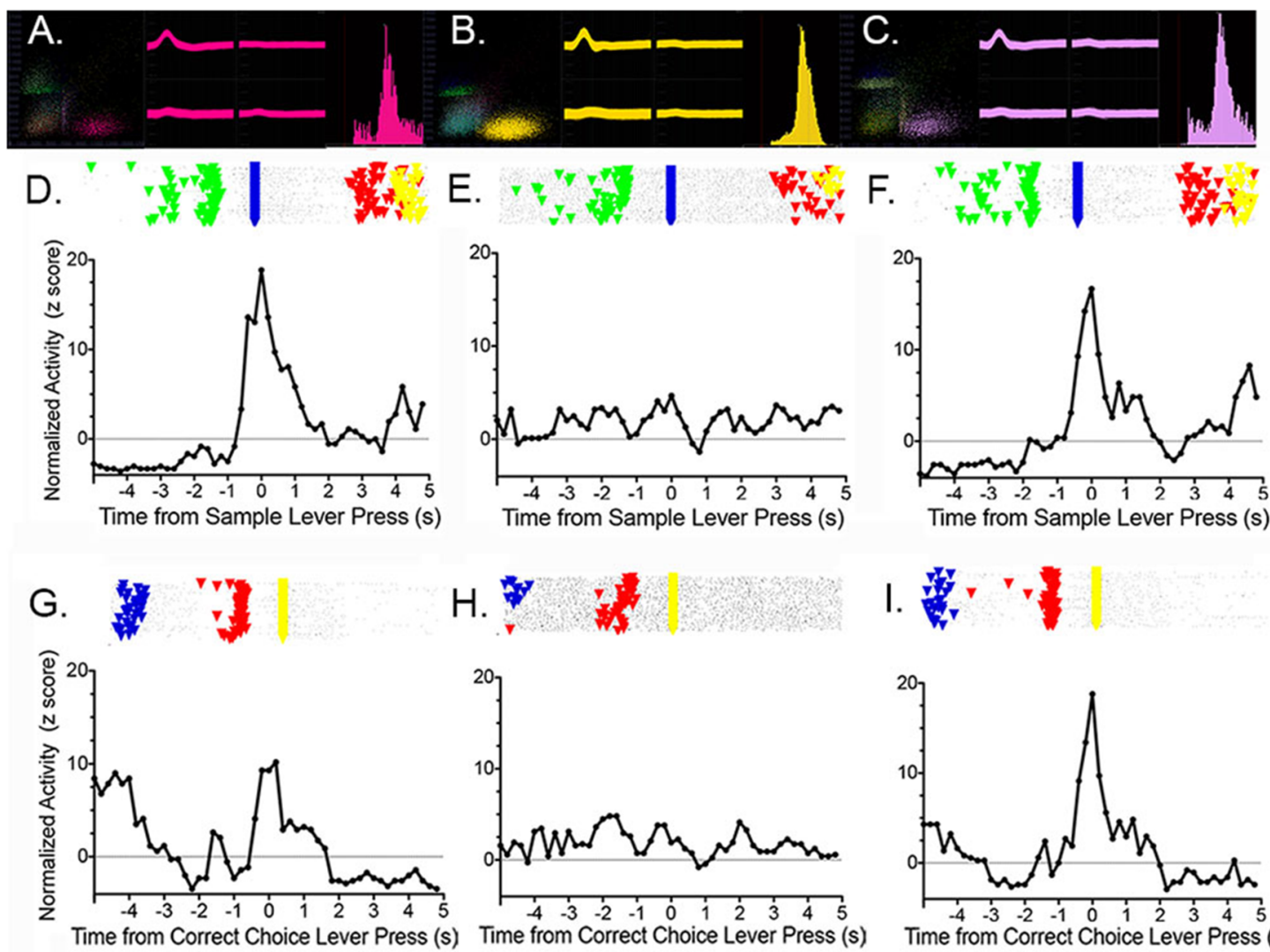

Time from Correct Choice Lever Press (s)
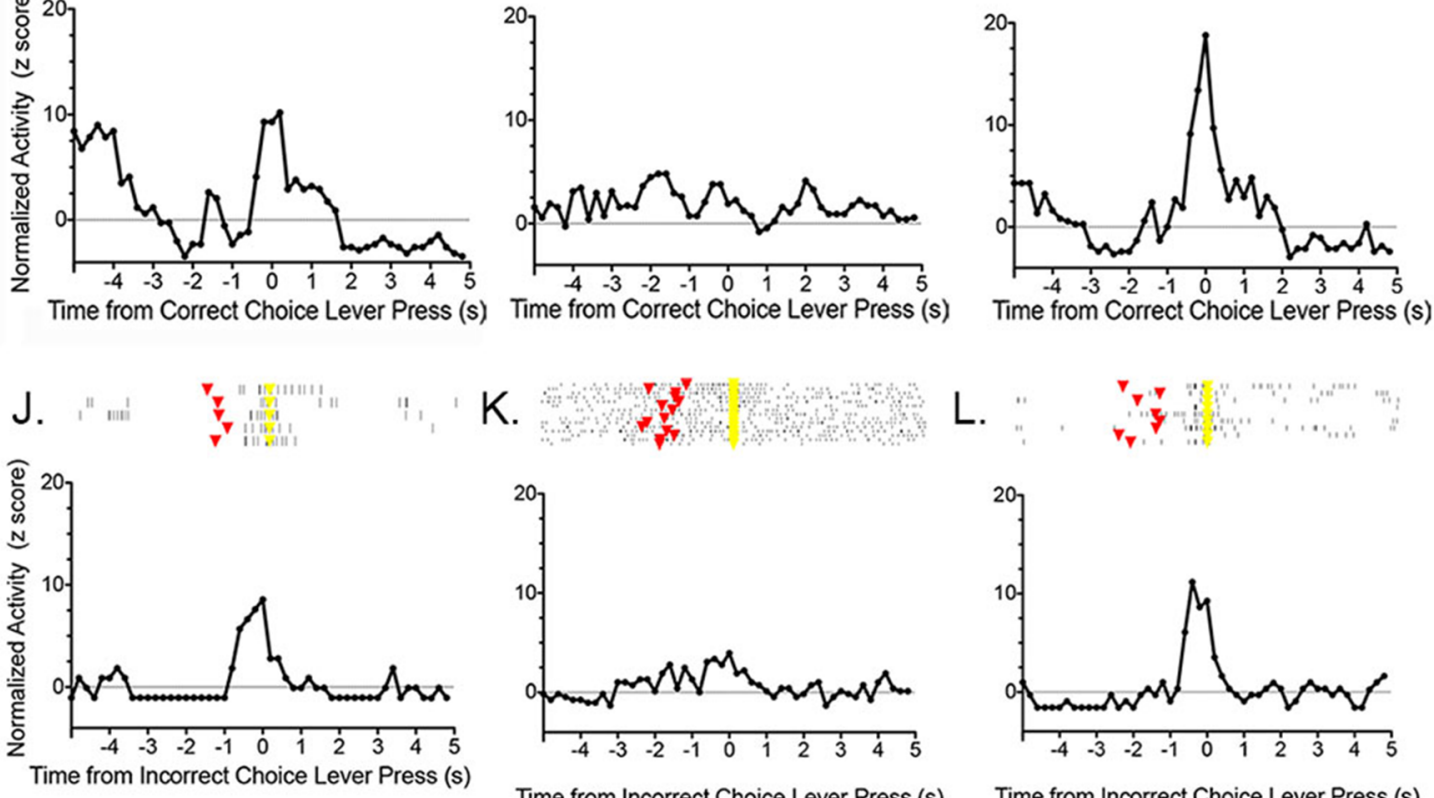

K.
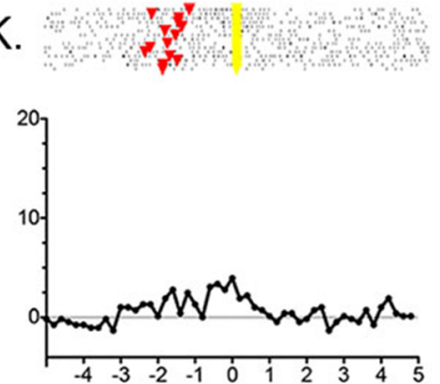

Time from Incorrect Choice Lever Press (s)
L.
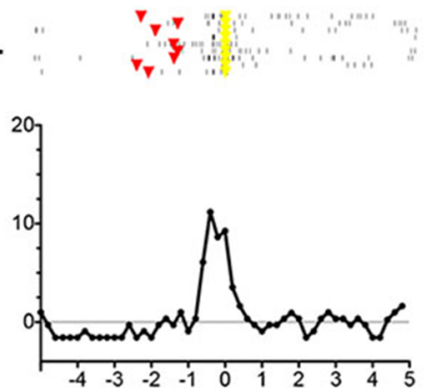

Time from Incorrect Choice Lever Press (s)

FIGURE 13 | Effects of unilateral thalamic inactivation by $1.0 \mathrm{nmol}$ muscimol near the junction of the paracentral and mediodorsal nuclei on a reinforcement anticipation (RA) response in ipsilateral mPFC. Results are shown for pre-inactivation day 1 (A,D, G,J), inactivation day 2 (B,E,H,K), and post-inactivation day 3 (C,F,I,L). 3D cluster plots, waveforms at each tetrode wire, and the interspike interval histograms (A,B,C) confirm the identity of the neuron held across 3 days and show the increase in activity observed during thalamic inactivation. Rasters and normalized PETHs aligned with sample (D,E,F), correct choice (G,H,I), and incorrect choice (J,K,L) reveal typical RA responses on days 1 and 3 that disappear during day 2 thalamic inactivation. Figure reproduced from Francoeur et al. (2019).

(b) Rostral intralaminar and VM nuclei affect speed and accuracy of responding based on learned conditional rules, effects consistent with their prominent connections with striatum and motor cortices, respectively. Like mPFC lesions rostral intralaminar and VM lesions produce delay-independent impairments affecting response speed and accuracy for egocentric DMTP and DNMTP tasks while sparing allocentric DNMTP. (c) Anterior thalamic and ventral midline $\mathrm{Re}$ and $\mathrm{Rh}$ nuclei affect allocentric spatial function, consistent with their prominent connections with the hippocampal system. Anterior thalamic lesions spare egocentric DMTP and DNMTP tasks affected by mPFC lesions. ReRh lesions affect tasks that depend on both mPFC and hippocampus.

(d) Dorsal midline nuclei integrate inputs from visceral-, arousal-, and emotion-related areas of the brain and 
influence cortical and subcortical circuits related to mPFC function. They are important for behavioralstate control of adaptive responding and response to salient stimuli in associative learning. Dorsal midline lesions spare DMTP and DNMTP tasks that depend on mPFC.

4. During the dDNMTP task, most MD neurons with criterion event-related responses (237/254) exhibit temporal patterns of firing that closely match response types in mPFC. A preponderance of these are movement and reinforcementrelated responses critical for dDNMTP choice. MD lesions made before initial training selectively decrease the number of movement-related responses in mPFC.

5. Drug inactivation of MD and adjacent intralaminar nuclei broadly suppresses the expression of event-related activity in mPFC during the dDNMTP task. Optogenetic studies suggest that $\mathrm{MD}$ amplifies and sustains behaviorallyrelevant information in the $\mathrm{PFC}$, a process that might

\section{REFERENCES}

Aggleton, J. P., and Brown, M. W. (2006). Interleaving brain systems for episodic and recognition memory. Trends Cogn. Sci. 10, 455-463. doi: 10.1016/j.tics. 2006.08.003

Aggleton, J. P., and Nelson, A. J. D. (2015). Why do lesions in the rodent anterior thalamic nuclei cause such severe spatial deficits? Neurosci. Biobehav. Rev. 54, 131-144. doi: 10.1016/j.neubiorev.2014.08.013

Ahrens, A. M., Meyer, P. J., Ferguson, L. M., Robinson, T. E., and Aldridge, J. W. (2016). Neural activity in the ventral pallidum encodes variation in the incentive value of a reward cue. J. Neurosci. 36, 7957-7970. doi: 10.1523/JNEUROSCI.0736-16.2016

Alcaraz, F., Fresno, V., Marchand, A. R., Kremer, E. J., Cloutureau, E., and Wolff, M. (2018). Thalamocortical and cortricothalamic pathways differentially contribute to goal-directed behaviors in the rat. eLife 7:e32517. doi: 10.7554/eLife.32517

Alcaraz, F., Naneix, F., Desfosses, E., Marchand, A. R., Wolff, M., and Coutureau, E. (2016). Dissociable effects of anterior and mediodorsal thalamic lesions on spatial goal-directed behavior. Brain Struct. Funct. 221, 79-89. doi: 10.1007/s00429-014-0893-7

Amiez, C., Joseph, J. P., and Procyk, E. (2006). Reward encoding in the monkey anterior cingulate cortex. Cereb. Cortex 16, 1040-1055. doi: 10.1093/cercor/bhj046

Baeg, E. H., Kim, Y. B., Huh, K., Mook-Jung, I., Kim, H. T., and Jung, M. W. (2003). Dynamics of population code for working memory in the prefrontal cortex. Neuron 40, 177-188. doi: 10.1016/s0896-6273(03)00597-x

Bailey, K. R., and Mair, R. G. (2004). Dissociable effects of frontal cortical lesions on measures of visuospatial attention and spatial working memory in the rat. Cereb. Cortex 9, 974-985. doi: 10.1093/cercor/bhh058

Bailey, K. R., and Mair, R. G. (2005). Lesions of specific and nonspecific thalamic nuclei affect prefrontal cortex-dependent aspects of spatial working memory. Behav. Neurosci. 119, 410-419. doi: 10.1037/0735-7044. 119.2.410

Bailey, K. R., and Mair, R. G. (2006). The role of striatum in initiation and execution of learned action sequences in rats. J. Neurosci. 26, 1016-1025. doi: 10.1523/JNEUROSCI.3883-05.2006

Bailey, K. R., and Mair, R. G. (2007). Effects of frontal cortex lesions on action sequence learning in the rat. Eur. J. Neurosci. 25, 2905-2915. doi: 10.1111/j. 1460-9568.2007.05492.x

Balleine, B. W. (2019). The meaning of behavior: discriminating reflex and volition in the brain. Neuron 104, 47-62. doi: 10.1016/j.neuron.2019. 09.024

Balleine, B. W., and Dickinson, A. (1998). Goal-directed instrumental action: contingency and incentive learning and their cortical substrates. help tune mPFC neurons to respond to task-relevant information during goal-directed behavior or suppress the expression of event-related activity during more prolonged drug inactivation.

\section{AUTHOR CONTRIBUTIONS}

RM was primarily responsible for writing the article. MF and BG contributed to writing and discussion of the manuscript. All authors contributed to the article and approved the submitted version.

\section{FUNDING}

The work described here was supported by a Research Leveraging Initiative grant from the University of New Hampshire, MH110876 from National Institute of Mental Health (NIMH), and NS26855 from NINDS.

Neuropharmacology 37, 407-419. doi: 10.1016/s0028-3908(98) 00033-1

Balleine, B., and O’Doherty, J. (2010). Human and rodent homologies in action control: corticostriatal determinants of goal-directed and habitual action. Neuropsychopharmacology 35, 48-69. doi: 10.1038/npp.2009.131

Barbas, H., Henion, T. H. H., and Dermon, C. R. (1991). Diverse thalamic projections to the prefrontal cortex in the rhesus monkey. J. Comp. Neurol. 313, 65-94. doi: 10.1002/cne.903130106

Barson, J. R., Mack, N. R., and Gao, W.-J. (2020). The paraventricular nucleus of the thalamus is an important node in the emotional processing network. Front. Behav. Neurosci. 14:598469. doi: 10.3389/fnbeh.2020.598469

Bayer, L., Eggermann, E., Saint-Mleux, B., Machard, D., Jones, B. E., Mühlethaler, M., et al. (2002). Selective action of orexin (hypocretin) on nonspecific thalamocortical projection neurons. J. Neurosci. 22, 7835-7839. doi: 10.1523/JNEUROSCI.22-18-07835.2002

Becker, J. T., Walker, J. A., and Olton, D. (1980). Neuroanatomical bases of spatial memory. Brain Res. 200, 307-320. doi: 10.1016/0006-8993(80)90922-1

Berendse, H. W., and Groenewegen, H. J. (1990). Organization of the thalamostriatal projections in the rat, with special emphasis on the ventral striatum. J. Comp. Neurol. 299, 187-228. doi: 10.1002/cne.902990206

Berendse, H. W., and Groenewegen, H. J. (1991). Restricted cortical termination fields of the midline and intralaminar thalamic nuclei in the rat. Neuroscience 42, 73-102. doi: 10.1016/0306-4522(91)90151-d

Birrell, J. M., and Brown, V. J. (2000). Medial frontal cortex mediates perceptual attention set shifting in the rat. J. Neurosci. 20, 4320-4324 doi: 10.1523/JNEUROSCI.20-11-04320.2000

Bolkan, S. S., Stujenske, J. M., Parnaudeau, S., Spellman, T. J., Rauffenbart, C., Abbas, A. I., et al. (2017). Thalamic projections sustain prefrontal activity during working memory maintenance. Nat. Neurosci. 20, 987-996. doi: $10.1038 / \mathrm{nn} .4568$

Bradfield, L. A., Hart, G., and Balleine, B. W. (2013). The role of the anterior, mediodorsal, and parafascicular thalamus in instrumental conditioning. Front Syst. Neurosci. 7:51. doi: 10.3389/fnsys.2013.00051

Brown, V. J., Bowman, E. M., and Robbins, T. W. (1991). Response-related deficits following unilateral lesions of medial agranular cortex of the rat. Behav. Neurosci. 105, 567-578. doi: 10.1037/0735-7044.105.4.567

Bubb, E. J., Aggleton, J. P., O’Mara, S. M., and Nelson, A. J. D. (2021). Chemogenetics reveal an anterior cingulate-thalamic pathway for attending to task-relevant information. Cereb. Cortex 31, 2169-2186. doi: 10.1093/cercor/bhaa353

Buchsbaum, B. R., and D’Esposito, M. D. (2019). A sensorimotor view of verbal working memory. Cortex 112, 134-148. doi: 10.1016/j.cortex.2018.11.010

Burk, J. A., and Mair, R. G. (1998). Thalamic amnesia reconsidered: excitotoxic lesions of the intralaminar nuclei, but not the mediodorsal nucleus, disrupt 
place delayed matching-to-sample performance in rats (Rattus norvegicus). Behav. Neurosci. 112, 54-67. doi: 10.1037/0735-7044.112.1.54

Burk, J. A., and Mair, R. G. (1999). Delayed matching-to-sample trained with retractable levers is impaired by lesions of the intralaminar or ventromedial but not the laterodorsal thalamic nuclei. Psychobiology 27, 351-363. doi: 10.3758/BF03332128

Burk, J. A., and Mair, R. G. (2001a). Effects of dorsal and ventral striatal lesions on delayed matching trained with retractable levers. Behav. Brain Res. 122, 67-78. doi: 10.1016/s0166-4328(01)00169-3

Burk, J. A., and Mair, R. G. (2001b). Effects of intralaminar thalamic lesions on sensory attention and motor intention in the rat: a comparison with lesions involving frontal cortex and hippocampus. Behav. Brain Res. 123, 49-63. doi: 10.1016/s0166-4328(01)00202-9

Bussey, T. J., Muir, J. L., Everitt, B. J., and Robbins, T. W. (1997). Triple dissociation of anterior cingulate, posterior cingulate, and medial frontal cortices on visual discrimination tasks using a touchscreen testing procedure for the rat. Behav. Neurosci. 111, 920-936. doi: 10.1037/0735-7044. 111.5.920

Carlesimo, G. A., Lombardi, M. G., and Caltagirone, C. (2011). Vascular thalamic amnesia: a reappraisal. Neuropsychologia 49, 777-789. doi: 10.1016/j. neuropsychologia.2011.01.026

Cavanaugh, S. E., Hunt, L. T., and Kennerley, S. W. (2020). A diversity of intrinsic timescales underlie neural computations. Front. Neural Circuits 14:615626. doi: $10.3389 /$ fncir.2020.615626

Chang, J.-Y., Chen, L., Luo, F., Shi, L.-H., and Woodward, D. J. (2002). Neuronal responses in the frontal cortico-basal ganglia system during delayed matchingto-sample task: ensemble recording in freely moving rats. Exp. Brain Res. 142, 67-80. doi: 10.1007/s00221-001-0918-3

Chudasama, Y. (2011). Animal models of prefrontal-executive function. Behav. Neurosci. 125, 327-343. doi: 10.1037/a0023766

Chudasama, Y., and Muir, J. L. (2001). Visual attention in the rat: a role or the prelimbic cortex and thalamic nuclei? Behav. Neurosci. 115, 417-428. doi: 10.1037/0735-7044.115.2.417

Chudasama, Y., Passetti, F., Rhodes, S. E. V., Lopian, R. D., Desai, A., and Robbins, T. W. (2003). Dissociable aspects of performance on the 5-choice serial reaction time task following lesions of the dorsal anterior cingulate, infralimbic and orbitofrontal cortex in the rat: differential effects on selectivity, impulsivity and compulsivity. Behav. Brain Res. 146, 105-119. doi: 10.1016/j. bbr.2003.09.020

Collins, D. P., Anastasiades, P. G., Marlin, J. J., and Carter, A. G. (2018). Reciprocal circuits linking the prefrontal cortex with dorsal and ventral thalamic nuclei. Neuron 98, 366-379. doi: 10.1016/j.neuron.2018.03.024

Corbit, L. H., and Balleine, B. W. (2003). The role of prelimbic cortex in instrumental conditioning. Behav. Brain Res. 146, 145-157. doi: 10.1016/j.bbr. 2003.09.023

Costello, M. G., Zhu, D., May, P. J., Salinas, E., and Stanford, T. R. (2016). Task dependence of decision and choice-related activity in monkey oculomotor thalamus. J. Neurophysiol. 115, 581-601. doi: 10.1152/jn.00592.2015

Courtiol, E., and Wilson, D. A. (2016). Neural representation of odorguided behavior in the rat olfactory thalamus. J. Neurosci. 36, 5946-5960. doi: 10.1523/JNEUROSCI.0533-16.2016

Cowen, S. L., and McNaughton, B. L. (2007). Selective delay activity in the medial prefrontal cortex of the rat: contribution of sensorimotor information and contingency. J. Neurophysiol. 98, 303-316. doi: 10.1152/jn.001 50.2007

Cruikshank, S. J., Ahmed, O. J., Stevens, T. R., Patrick, S. L., Gonzalez, A. N., Elmaleh, M., et al. (2012). Thalamic control of layer 1 circuits in prefrontal cortex. J. Neurosci. 32, 17813-17823. doi: 10.1523/JNEUROSCI.323112.2012

Dalley, J., Cardinal, R., and Robbins, T. (2004). Prefrontal executive and cognitive functions in rodents: neural and neurochemical substrates. Neurosci. Biobehav. Rev. 28, 771-784. doi: 10.1016/j.neubiorev.2004.09.006

de Bruin, J. P., Moita, M. P., Brabander, H. M., and Joosten, R. N. (2001). Place and response learning of rats in a morris water maze: differential effects of fimbria fornix and medial prefrontal cortex lesions. Neurobiol. Learn. Mem. 75, 164-178. doi: 10.1006/nlme.2000.3962

de Saint Blanquat, P., Hok, V., Alvernhe, A., Save, E., and Poucet, B. (2010). Tagging items in spatial working memory: a unit-recording study in the rat medial prefrontal cortex. Behav. Brain Res. 209, 267-273. doi: 10.1016/j.bbr. 2010.02.004

Di Russo, F., Berchicci, M., Bozzacchi, C., Perri, R. L., Pitzalis, S., and Spinelli, D. (2017). Beyond the "Bereitschaftspotential": action preparation behind cognitive functions. Neurosci. Biobehav. Rev. 789, 57-81. doi: 10.1016/j. neubiorev.2017.04.019

Divac, I. (1971). Frontal lobe system and spatial reversal in the rat. Neuropsychologia 9, 175-183. doi: 10.1016/0028-3932(71)90041-8

Dolleman-van der Weel, M. J., Griffin, A. L., Ito, H. T., Shapiro, M. L., Witter, M. P., Vertes, R. P., et al. (2019). The nucleus reuniens of the thalamus sits at the nexus of a hippocampus and medial prefrontal cortex circuit enabling memory and behavior. Learn. Mem. 26, 191-205. doi: 10.1101/lm.048389.118

Domenech, P., Rheims, S., and Koechlin, E. (2020). Neural mechanisms resolving exploitation-exploration dilemmas in the medial prefrontal cortex. Science 369, 1056-1057. doi: 10.1126/science.abb0184

Dunnett, S. B. (1990). Role of prefrontal cortex and stratal output systems in short-term memory deficits associated with aging, basal forebrain lesions, and cholinergic-rich grafts. Can. J. Psychol. 44, 210-232. doi: 10.1037/h0084240

Enel, P., Wallis, J. D., and Rich, E. L. (2020). Stable and dynamic representations of value in the prefrontal cortex. eLife 9:e54313. doi: 10.7554/eLife 54313

Ennaceur, A., Neave, N., and Aggleton, J. P. (1997). Spontaneous object recognition and object location memory in rats: the effects of lesions in the cingulate cortices, the medial prefrontal cortex, the cingulum bundle and the fornix. Exp. Brain Res. 113, 509-519. doi: 10.1007/pl00005603

Euston, D. R., Gruber, A. J., and McNaughton, B. L. (2012). The role of medial prefrontal cortex in memory and decision making. Neuron 76, 1057-1070. doi: 10.1016/j.neuron.2012.12.002

Euston, D. R., and McNaughton, B. L. (2006). Apparent encoding of sequential context in rat medial prefrontal cortex is accounted for by behavioral variability. J. Neurosci. 26, 13143-13155. doi: 10.1523/JNEUROSCI.380306.2006

Fisher, B. M., Saksida, L. M., Robbins, T. W., and Bussey, T. J. (2020). Functional dissociations between subregions of the medial prefrontal cortex on the rodent touchscreen continuous performance test (rCPT) of attention. Behav. Neurosci. 134, 1-14. doi: 10.1037/bne0000338

Floresco, S. B., Braaksma, D. N., and Phillips, A. G. (1999). Thalamic-corticalstriatal circuitry subserves working memory during delayed responding on a radial arm maze. J. Neurosci. 19, 11061-11071. doi: 10.1523/JNEUROSCI.1924-11061.1999

Francoeur, M. J. (2019). The Cognitive Thalamus: Influence on Prefrontal Cortex and Executive Function. [Dissertation]. Durham, NH: University of New Hampshire.

Francoeur, M. J., and Mair, R. G. (2018). Representation of actions and outcomes in medial prefrontal cortex during delayed conditional decision making: population analyses of single neuron activity. Brain Neurosci. Adv. 2, 1-15. doi: $10.1177 / 2398212818773865$

Francoeur, M. J., and Mair, R. G. (2020). Effects of choice on neuronal activity in anterior cingulate, prelimbic, and infralimbic cortices in the rat: comparison of serial lever pressing with delayed nonmatching to position. Eur. J. Neurosci. 51, 2052-2069. doi: 10.1111/ejn.14643

Francoeur, M. J., Wormwood, B. A., Gibson, B. M., and Mair, R. G. (2019). Central thalamic inactivation impairs the expression of action- and outcome-related responses in medial prefrontal cortex neurons in the rat. Eur. J. Neurosci. 50, 1779-1798. doi: 10.111/ejn.14350

Fresno, V., Parkes, S. L., Faugère, A., Coutureau, E., and Wolff, M. (2019). A thalamocortical circuit for updating action-outcome associations. eLife 23:e46187. doi: 10.7554/eLife.46187

Fuster, J. M. (2001). The prefrontal cortex - an update: time is of the essence. Neuron 30, 319-333. doi: 10.1016/s0896-6273(01)00285-9

Fuster, J. M., and Alexander, G. E. (1971). Neuron activity related to short-term memory. Science 173, 652-654. doi: 10.1126/science.173.3997.652

Georgopoulos, A. P., and Carpenter, A. F. (2015). Coding of movements in the motor cortex. Curr. Opin. Neurobiol. 33, 34-39. doi: 10.1016/j.conb.2015. 01.012

Germann, J., and Petrides, M. (2020). Area 8A within the posterior medial frontal gyrus underlies cognitive selection between competing visual targets. eNeuro 7:ENEURO.0102-20.2020. doi: 10.1523/ENEURO.0102-20.2020 
Goldman, P. S., and Rosvold, H. E. (1970). Localization of function within the dorsolateral prefrontal cortex of the rhesus monkey. Exp. Neurol. 27, 291-304. doi: 10.1016/0014-4886(70)90222-0

Goldman, P. S., Rosvold, H. E., Vest, B., and Galkin, T. W. (1971). Analysis of the delayed-alternation deficit produced by dorsolateral prefrontal lesions in the rhesus monkey. J. Comp. Physiol. Psychol. 77, 212-220. doi: 10.1037/h00 31649

Goldman-Rakic, P. S. (1996). The prefrontal landscape: implications of functional architecture for understanding human mentation and the central executive. Philos. Trans. R. Soc. Lond. B Biol. Sci. 351, 1445-1453. doi: 10.1098/rstb. 1996.0129

Goldman-Rakic, P. S. (2005). Cellular basis of working memory. Neuron 14, 477-485. doi: 10.1016/0896-6273(95)90304-6

Goldman-Rakic, P. S., and Selemon, L. D. (1997). Functional and anatomical aspects of prefrontal pathology in schizophrenia. Schizophr. Bull. 23, 437-458. doi: $10.1093 /$ schbul/23.3.437

Grillner, S., Hellgren, J., Ménard, A., Saitoh, K., and Wikström, M. A. (2005). Mechanisms for selection of basic motor programs - roles for the striatum and pallidum. Trends Neurosci. 28, 364-370. doi: 10.1016/j.tins.2005.05.004

Groenewegen, H. J. (1988). Organization of the afferent connections of the mediodorsal thalamic nucleus in the rat, related to the mediodorsal prefrontal topography. Neuroscience 24, 379-431. doi: 10.1016/0306-4522(88) 90339-9

Groenewegen, H. J., and Berendse, H. W. (1994). The specificity of the 'nonspecific' midline and intralaminar thalamic nuclei. Trends Neurosci. 17, 52-57. doi: 10.1016/0166-2236(94)90074-4

Guo, Z. V., Inagaki, H. K., Daie, K., Druckmann, S., Gerfen, C. R., and Svoboda, K. (2017). Maintenance of persistent firing in a frontal thalamocortical loop. Nature 545, 181-186. doi: 10.1038/nature22324

Haight, J. L., Fraser, K. M., Akil, H., and Flagel, S. B. (2015). Lesions of the paraventricular nucleus of the thalamus differentially affect signand goal-tracking conditioned responses. Eur. J. Neurosci. 42, 2478-2488. doi: 10.1111/ejn.13031

Halassa, M. M., and Sherman, S. M. (2019). Thalamocortical circuit motifs: a general framework. Neuron 103, 762-770. doi: 10.1016/j.neuron.2019.06.005

Han, J., Lee, J. H., Kim, M. J., and Jung, M. W. (2013). Neural activity in mediodorsal nucleus of thalamus in rats performing a working memory task. Front. Neural Circuits 7:128. doi: 10.3389/fncir.2013.00128

Harrison, L. M., and Mair, R. G. (1996). A comparison of the effects of frontal cortical and thalamic lesions on measures of spatial learning and memory in the rat. Behav. Brain Res. 75, 195-206. doi: 10.1016/0166-4328(96) 00173-8

Hart, G., and Balleine, B. W. (2016). Consolidation of goal-directed action depends on MAPK/ERK signaling in rodent prelimbic cortex. J. Neurosci. 36, 11974-11986. doi: 10.1523/JNEUROSCI.1772-16.2016

Hart, E. E., Blair, G. J., O’Dell, T. J., Blair, H. T., and Izquierdo, A. (2020). Chemogenetic modulation and single-photon calcium imaging in anterior cingulate cortex reveal a mechanism for effort-based decisions. J. Neurosci. 40, 5628-5643. doi: 10.1523/JNEUROSCI.2548-19.2020

Heidbreder, C. A., and Groenewegen, H. J. (2003). The medial prefrontal cortex in the rat: evidence for a dorso-ventral distinction based on functional and anatomical characteristics. Neurosci. Biobehav. Rev. 27, 555-579. doi: 10.1016/j. neubiorev.2003.09.003

Hembrook, J. R., and Mair, R. G. (2011). Lesions of reuniens and rhomboid thalamic nuclei impair radial maze win-shift performance. Hippocampus 21, 815-826. doi: 10.1002/hipo.20797

Hembrook, J. R., Onos, K. D., and Mair, R. G. (2012). Inactivation of ventral midline thalamus produces selective spatial delayed conditional discrimination impairment in the rat. Hippocampus 22, 853-860. doi: 10.1002/hipo. 20945

Hernández, M. C., Navarro-Castilla, Á., Wilsterman, K., Bentley, G. E., and Barja, I. (2019). When food access is challenging: evidence of wood mice ability to balance energy budget under predation risk and physiological stress reactions. Behav. Ecol. Sociobiol. 73:145. doi: 10.1007/s00265-0192756-y

Hok, V., Save, E., Lenck-Santini, P. P., and Poucet, B. (2005). Coding for spatial goals in the prelimbic/infralimbic area of the rat frontal cortex. Proc. Natl. Acad. Sci. U S A 102, 4602-4607. doi: 10.1073/pnas.0407332102
Hoover, W. B., and Vertes, R. P. (2007). Anatomical analysis of afferent projections to the medial prefrontal cortex in the rat. Brain Struct. Funct. 212, 149-179. doi: 10.1007/s00429-007-0150-4

Horst, N. K., and Laubach, M. (2012). Working with memory: evidence for a role for the medial prefrontal cortex in performance monitoring during spatial delayed alternation. J. Neurophysiol. 108, 3276-3288. doi: 10.1152/jn. 01192.2011

Hunt, P. R., and Aggleton, J. P. (1998). Neurotoxic lesions of ther dorsomedial thalamus impair the acquisition but not the performance of delayed matching to place by rats: a deficit in shifting response rules. J. Neurosci. 18, 10045-10052. doi: 10.1016/s0166-4328(98)00033-3

Huo, Y., Chen, H., and Guo, Z. V. (2020). Mapping functional connectivity from the dorsal cortex to the thalamus. Neuron 107, 1080-1094. doi: 10.1016/j. neuron.2020.06.038

Hyman, J. M., Ma, L., Balaguer-Ballester, E., Durstewitz, D., and Seamans, J. K. (2012). Contextual encoding by ensembles of medial prefrontal cortex neurons. Proc. Natl. Acad. Sci. U S A 109, 5086-5091. doi: 10.1073/pnas.11144 15109

Hyman, J. M., Whitman, J., Emberly, E., Woodward, T. S., and Seamans, J. K. (2013). Action and outcome activity state patterns in the anterior cingulate cortex. Cereb. Cortex 23, 1257-1268. doi: 10.1093/cercor/bhs104

Insel, N., and Barnes, C. A. (2015). Differential activation of fast-spiking and regular-firing neuron populations during movement and reward in the dorsal medial frontal cortex. Cereb. Cortex 25, 2631-2647. doi: 10.1093/cercor/ bhu062

Isseroff, A., Rosvold, H. E., Galkin, T. W., and Goldman-Rakic, P. S. (1982). Spatial memory impairments following damage to the mediodorsal nucleus of the thalamus in rhesus monkeys. Brain Res. 232, 97-113. doi: 10.1016/00068993(82)90613-8

Jacobsen, C. F. (1936). The functions of the frontal association areas in monkeys. Comp. Psychol. Monographs 13, 1-60.

Jung, M. W., Qin, Y., McNaughton, B. L., and Barnes, C. A. (1998). Firing characteristics of deep layer neurons in prefrontal cortex in rats performing spatial working memory tasks. Cereb. Cortex 8, 437-450. doi: 10.1093/cercor/8. 5.437

Kakei, S., Hoffman, D. S., and Strick, P. L. (2001). Direction of action is represented in the ventral premotor cortex. Nat. Neurosci. 10, 1020-1025. doi: $10.1038 / \mathrm{nn} 726$

Kakei, S., Hoffman, D. S., and Strick, P. L. (2003). Sensorimotor transformations in cortical motor areas. Neurosci. Res. 46, 1-10. doi: 10.1016/s01680102(03)00031-2

Kennerley, S. W., Sakai, K., and Rushworth, M. F. S. (2004). Organization of action sequences and the role of the pre-SMA. J. Neurophysiol. 91, 978-993. doi: 10.1152/jn.00651.2003

Kesner, R. P., and Churchwell, J. C. (2011). An analysis of rat prefrontal cortex in mediating executive function. Neurobiol. Learn. Mem. 96, 417-431. doi: 10.1016/j.nlm.2011.07.002

Kesner, R. P., Hunt, M. E., Williams, J. M., and Long, J. M. (1996). Prefrontal cortex and working memory for spatial response, spatial location, and visual object information in the rat. Cereb. Cortex 6, 311-318. doi: 10.1093/cercor/ 6.2 .311

Kinomura, S., Larsson, J., Gulyás, B., and Roland, P. E. (1996). Activation by attention of the human. Reticular formation and thalamic intralaminar nuclei. Science 271, 512-515. doi: 10.1126/science.271.5248.512

Kirouac, G. J. (2015). Placing the paraventricular nucleus of the thalamus within the brain circuits that control behavior. Neurosci. Biobehav. Rev. 56, 315-329. doi: 10.1016/j.neubiorev.2015.08.005

Koger, S. M., and Mair, R. G. (1994). Comparison of the effects of frontal cortical and thalamic lesions on measures of olfactory learning and memory in the rat. Behav. Neurosci. 108, 1088-1100. doi: 10.1037/0735-7044.108. 6.1088

Kolb, B., Nonneman, A. J., and Singh, R. K. (1974). Double dissociation of spatial impairments and perseveration following selective prefrontal lesions in rats. J. Comp. Physiol. Psychol. 87, 772-780. doi: 10.1037/h0036970

Kolb, B., Sutherland, R. J., and Whishaw, I. Q. (1983). A comparison of the contributions of the frontal and parietal association cortex to spatial localization in rats. Behav. Neurosci. 97, 13-27. doi: 10.1037/0735-7044. 97.1 .13 
Krell, E. (2020). Neurons in Ventral Pallidum Respond Selectively to Reward and Related Events During Reward-Guided Decision Making. [Masters thesis]. Durham, NH: University of New Hampshire.

Krout, K. E., Belzer, R. E., and Loewy, A. D. (2002). Brainstem projections to midline and intralaminar thalamic nuclei of the rat. J. Comp. Neurol. 448, 53-101. doi: 10.1002/cne.10236

Krout, K. E., and Loewy, A. D. (2000a). Periaqueductal gray matter projections to midline and intralaminar thalamic nuclei in the rat. J. Comp. Physiol. 424, 111-141. doi: 10.1002/1096-9861(20000814)424:1 $<111:$ aid-cne9>3.0.co;2-3

Krout, K. E., and Loewy, A. D. (2000b). Parabrachial nucleus projections to midline and intralaminar nuclei of the rat. J. Comp. Neurol. 428, 475-494. doi: 10.1002/1096-9861(20001218)428:3<475::aid-cne6 >3.0.co;2-9

Krout, K. E., Loewy, A. D., Westby, G. W., and Redgrave, P. (2001). Superior colliculus projections to midline and intralaminar nuclei of the rat. J. Comp. Neurol. 431, 198-216. doi: 10.1002/1096-9861(20010305)431:2<198::aidcne1065>3.0.co;2-8

Kuramoto, E., Pan, S., Furuta, T., Tanaka, Y., Iwai, H., Yamanaka, A., et al. (2017). Individual mediodorsal thalamic neurons project to multiple areas of the rat prefrontal cortex: a single neuron-tracing study using virus vectors. J. Comp. Neurol. 525, 166-185. doi: 10.1002/cne.24054

Larsen, J. K., and Divac, I. (1978). Selective ablations within the prefrontal cortex of the rat and performance of delayed alternation. Physiol. Psychol. 6, 15-17. doi: $10.3758 / b f 03326684$

Lee, J., Dong, S., Jeong, J., and Yoon, B. (2020). Effects of transcranial direct current stimulation over the dorsolateral prefrontal cortex (PFC) on cognitive-motor dual control skills. Percept. Mot. Skills 127, 803-822. doi: $10.1177 / 0031512520935695$

Lilly, M. V., Lucore, E. C., and Tarvin, K. A. (2019). Eavesdropping grey squirrels infer safety from bird chatter. PLoS One 14:e0221279. doi: 10.1371/journal. pone. 0221279

Magrath, R. D., Haff, T. M., Fallow, P. M., and Radford, A. N. (2015). Eavesdropping on heterospecific alarm calls: from mechanisms to consequences. Biol. Rev. 90, 560-586. doi: 10.1111/brv.12122

Mair, R. G. (1994). On the role of thalamic pathology in diencephalic amnesia. Rev. Neurosci. 5, 105-140. doi: 10.1515/revneuro.1994.5.2.105

Mair, R. G., Anderson, C. D., Langlais, P. J., and McEntee, W. J. (1988). Behavioral impairments, brain lesions and monoaminergic activity in the rat following recovery from a bout of thiamine deficiency. Behav. Brain Res. 27, 223-239. doi: 10.1016/0166-4328(88)90119-2

Mair, R. G., Burk, J. A., and Porter, M. C. (1998). Lesions of the frontal cortex, hippocampus, and intralaminar thalamic nuclei have distinct effects on remembering in rats. Behav. Neurosci. 112, 772-792. doi: 10.1037/0735-7044. 112.4.772

Mair, R. G., Burk, J. A., and Porter, M. C. (2003). Impairment of radial maze delayed nonmatching after lesions of anterior thalamus and parahippocampal cortex. Behav. Neurosci. 117, 596-605. doi: 10.1037/0735-7044.117. 3.596

Mair, R. G., and Hembrook, J. R. (2008). Memory enhancement with eventrelated stimulation of the rostral intralaminar thalamic nuclei. J. Neurosci. 28, 14293-14300. doi: 10.1523/JNEUROSCI.3301-08.2008

Mair, R. G., Koch, J. K., Newman, J. B., Howard, J. R., and Burk, J. A. (2002). A double dissociation in striatum between serial reaction time and radial maze delayed nonmatching performance in rats. J. Neurosci. 22, 6756-6765. doi: 10.1523/JNEUROSCI.22-15-06756.2002

Mair, R. G., and Lacourse, D. M. (1992). Radio-frequency lesions of the thalamus produce delayed-nonmatching-to-sample impairments comparable to pyrithiamine-induced encephalopathy in rats. Behav. Neurosci. 106, 634-645. doi: 10.1037/0735-7044.106.4.634

Mair, R. G., Miller, R. L., Wormwood, B. A., Francoeur, M. J., Onos, K. D., and Gibson, B. M. (2015). The neurobiology of thalamic amnesia: contributions of medial thalamus and prefrontal cortex to delayed conditional discrimination. Neurosci. Biobehav. Rev. 54, 161-174. doi: 10.1016/j.neubiorev.2015. 01.011

Mair, R. G., Otto, T. A., Knoth, R. L., Rabchenuk, S. A., and Langlais, P. J. (1991). Analysis of aversively conditioned learning and memory in rats recovered from pyrithiamine-induced thiamine deficiency. Behav. Neurosci. 105, 351-359. doi: $10.1037 / 0735-7044.105 .3 .351$
Mannella, F., Mirolli, M., and Baldassarre, G. (2016). Goal-directed behavior and instrumental devaluation: a neural system-level computational model. Front. Behav. Neurosci. 10:181. doi: 10.3389/fnbeh.2016.00181

Marsh, B. T., Tarigoppula, V. S., Chen, C., and Francis, J. T. (2015). Toward an autonomous brain machine interface: integrating sensorimotor reward modulation and reinforcement learning. J. Neurosci. 35, 7374-7387. doi: 10.1523/JNEUROSCI.1802-14.2015

Marton, T., Seifikar, H., Luongo, F., Lee, A., and Sohal, V. (2018). Roles of prefrontal cortex and mediodorsal thalamus in task engagement and behavioral flexibility. J. Neurosci. 38, 2569-2578. doi: 10.1523/JNEUROSCI.1728-17.2018

Mathiasen, M. M., O'Mara, S. M., and Aggleton, J. P. (2020). The anterior thalamic nuclei and nucleus reuniens: so similar but so different. Neurosci. Biobehav Rev. 119, 268-280. doi: 10.1016/j.neubiorev.2020.10.006

Matsumoto, M., Matsumotyo, K., Abe, H., and Tanaka, K. (2007). Medial prefrontal cell activity signaling prediction errors of action values. Nat. Neurosci. 10, 647-656. doi: 10.1038/nn1890

McGinty, J. F., and Otis, J. M. (2020). Heterogeneity in the paraventricular thalamus: the traffic light of motivated behaviors. Front. Behav. Neurosci. 14:590528. doi: 10.3389/fnbeh.2020.590528

Meyer, T., Qi, X.-L., Stanford, T. R., and Constantinidis, C. (2011). Stimulus selectivity in dorsal and ventral prefrontal cortex after training in working memory tasks. J. Neurosci. 31, 6266-6276. doi: 10.1523/JNEUROSCI.679810.2011

Miller, E. K., and Cohen, J. D. (2001). An integrative theory of prefrontal cortex function. Annu. Rev. Neurosci. 24, 167-202. doi: 10.1146/annurev. neuro.24.1.167

Miller, R. L. A., Francoeur, M. J., Gibson, B. M., and Mair, R. G. (2017). Mediodorsal thalamic neurons mirror the activity of medial prefrontal neurons responding to movement and reinforcement during a dynamic DNMTP task. eNeuro 4:ENEURO.0196-17.2017. doi: 10.1523/ENEURO.019617.2017

Miller, E. K., Lundqvist, M., and Bastros, A. (2018). Working memory 2.0. Neuron 100, 463-475. doi: 10.1016/j.neuron.2018.09.023

Mitchell, A. S., and Chakraborty, S. (2013). What does the mediodorsal thalamus do? Front. Syst. Neurosci. 7:37. doi: 10.3389/fnsys.2013.00037

Mitchell, A. S., and Dalrymple-Alford, J. C. (2006). Lateral and anterior thalamic lesions impair independent memory systems. Learn. Mem. 13, 388-396. doi: 10.1101/lm.122206

Mitchell, A. S., Sherman, S. M., Sommer, M. A., Mair, R. G., Vertes, R. P., and Chudasama, Y. (2014). Advances in understanding mechanisms of thalamic relays in cognition and behavior. J. Neurosci. 34, 15340-15346. doi: 10.1523/JNEUROSCI.3289-14.2014

Muir, J. L., Everitt, B. J., and Robbins, T. W. (1996). The cerebral cortex of the rat and visual attentional function: dissociable effects of mediofrontal, cingulate, anterior dorsolateral, and parietal cortex on a five-choice serial reaction time task. Cereb. Cortex 6, 470-481. doi: 10.1093/cercor/6.3.470

Murakami, M., and Mainen, Z. F. (2015). Preparing and selecting actions with neural populations: toward cortical circuit mechanisms. Curr. Opin. Neurobiol. 33, 40-46. doi: 10.1016/j.conb.2015.01.005

Navarro-Castilla, A., Barja, I., and Díaz, M. (2018). Foraging, feeding, and physiological stress responses of wild wood mice to increased illumination and common genet cues. Curr. Zool. 64, 409-417. doi: 10.1093/cz/zox048

Newman, L. A., and Mair, R. G. (2007). Cholinergic modulation of visuospatial responding in central thalamus. Eur. J. Neurosci. 26, 3543-3552. doi: 10.1111/j. 1460-9568.2007.05961.x

Nonacs, P. (2001). State dependent behavior and the marginal value theorem. Behav. Ecol. 12, 71-83. doi: 10.1093/oxfordjournals.beheco.a0 00381

O'Mara, S., and Aggleton, J. (2019). Space and memory (far) beyond the hippocampus: many subcortical structures also support cognitive mapping and mnemonic processing. Front. Neural Circuits 13:52. doi: 10.3389/fncir.2019. 00052

Öngür, D., and Price, J. L. (2000). The organization of networks within the orbital and medial prefrontal cortex of rats, monkeys and humans. Cereb. Cortex 10, 206-219. doi: 10.1093/cercor/10.3.206

Onos, K. D., Francoeur, M. J., Wormwood, B. A., Miller, R. L. A., Gibson, B. M., and Mair, R. G. (2016). Prefrontal neurons encode actions and outcomes in conjunction with spatial location in rats performing a dynamic delayed 
non-match to position task. PLoS One 11:e0149019. doi: 10.1371/journal.pone. 0149019

Ostlund, S. B., and Balleine, B. W. (2005). Lesions of medial prefrontal cortex disrupt the acquisition but not the expression of goal-directed learning. J. Neurosci. 25, 7763-7770. doi: 10.1523/JNEUROSCI.1921-05.2005

Ostlund, S. B., and Balleine, B. W. (2008). Differential involvement of the basolateral amygdala and mediodorsal thalamus in instrumental action selection. J. Neurosci. 28, 4398-4405. doi: 10.1523/JNEUROSCI.5472-07.2008

Ottenheimer, D., Richard, J. M., and Janak, P. H. (2018). Ventral pallidum encodes relative reward value earlier and more robustly than nucleus accumbens. Nat. Commun. 9:4350. doi: 10.1038/s41467-018-06849-Z

Oyoshi, T., Nishio, H., Asakura, T., and Ono, T. (1996). Emotional and behavioral correlates of mediodorsal thalamic neurons during associative learning in rats. J. Neurosci. 16, 5812-5829. doi: 10.1523/JNEUROSCI.16-18-05812.1996

Parnaudeau, S., Bolkan, S. S., and Kellendonk, C. (2018). The mediodorsal thalamus: an essential partner of the prefrontal cortex for cognition. Biol. Psychiatry 83, 648-656. doi: 10.1016/j.biopsych.2017.11.008

Perrin, E., and Venance, L. (2019). Bridging the gap between striatal plasticity and learning. Curr. Opin. Neurobiol. 54, 104-112. doi: 10.1016/j.conb.2018. 09.007

Petrides, M. (1985). Deficits in non-spatial conditional associative learning after periarcuate lesions in the monkey. Behav. Brain Res. 16, 95-101. doi: 10.1016/0166-4328(85)90085-3

Poldrack, R. A., Sabb, F. W., Foerde, K., Tom, S. M., Asarnow, R. F., Bookheimer, S. Y., et al. (2005). The neural correlates of motor skill automaticity. J. Neurosci. 25, 5356-5364. doi: 10.1523/JNEUROSCI.388004.2005

Porter, M. C., Burk, J. A., and Mair, R. G. (2000). A comparison of the effects of hippocampal or prefrontal cortical lesions on three versions of delayed nonmatching-to-sample based on positional or spatial cues. Behav. Brain Res. 109, 69-81. doi: 10.1016/s0166-4328(99)00161-8

Porter, M. C., Koch, J., and Mair, R. G. (2001). Effects of reversible inactivation of thalamo-striatal circuitry on delayed matching trained with retractable levers. Behav. Brain Res. 119, 61-69. doi: 10.1016/s0166-4328(00)00331-4

Porter, M. C., and Mair, R. G. (1997). The effects of frontal cortical lesions on remembering depend on the procedural demands of tasks performed in the radial arm maze. Behav. Brain Res. 87, 115-125. doi: 10.1016/s01664328(96)02272-3

Powell, N. J., and Redish, A. D. (2014). Complex neural codes in rat prelimbic cortex are stable across days on a spatial decision task. Front. Behav. Neurosci. 8:120. doi: 10.3389/fnbeh.2014.00120

Prasad, J. A., MacGregor, E. M., and Chudasama, Y. (2013). Lesions of the thalamic reuniens cause impulsive but not compulsive responses. Brain Struct. Funct. 218, 85-96. doi: 10.1007/s00429-012-0378-5

Pratt, W. E., and Mizumori, S. J. (2001). Neurons in rat medial prefrontal cortex show anticipatory rate changes to predictable differential rewards in a spatial memory task. Behav. Brain Res. 123, 165-183. doi: 10.1016/s01664328(01)00204-2

Preuss, T. M. (1995). Do rats have prefrontal cortex? The Rose-Woolsey-Akert program reconsidered. J. Cogn. Neurosci. 7, 1-24. doi: 10.1162/jocn.1995. 7.1 .1

Ragazzino, M. E., Detrick, S., and Kesner, R. P. (2002). The effects of prelimbic and infralimbic lesions on working memory for visual objects in rats. Neurobiol. Learn. Mem. 77, 29-43. doi: 10.1006/nlme.2001.4003

Ragozzino, M. E., Adams, S., and Kesner, R. P. (1998). Differential involvement of the dorsal anterior cingulate and prelimbic-infralimbic areas of the rodent prefrontal cortex in spatial working memory. Behav. Neurosci. 112, 293-303. doi: 10.1037/0735-7044.112.2.293

Rajan, K., Harvey, C. D., and Tank, D. W. (2016). Recurrent network models of sequence generation and memory. Neuron 90, 128-142. doi: 10.1016/j.neuron. 2016.02.009

Ray, J. P., and Price, J. L. (1992). The organization of the thalamocortical connections of the mediodorsal thalamic nucleus in the rat, related to the ventral forebrain-prefrontal cortex topography. J. Comp. Neurol. 323, 167-197. doi: $10.1002 / \mathrm{cne} .903230204$

Reading, P. J., and Dunnett, S. B. (1991). The effects of excitotoxic lesions of nucleus accumbens on a matching to position task. Behav. Brain Res. 46, 17-29. doi: $10.1016 /$ s0166-4328(05)80093-2
Ren, S., Wang, Y., Yue, F., Cheng, X., Dang, R., Qiao, Q., et al. (2018). The paraventricular thalamus is a critical thalamic area for wakefulness. Science 362, 429-434. doi: 10.1126/science.aat 2512

Richard, J. M., Stout, N., Acs, D., and Janak, P. H. (2018). Ventral pallidal encoding of reward-seeking behavior depends on the underlying associative structure. eLife 7:e33107. doi: 10.7554/eLife.33107

Robinson, J. K., and Mair, R. G. (1992). MK-801 prevents brain lesions and delayed-nonmatching-to-sample deficits produced by pyrithiamine-induced encephalopathy in rats. Behav. Neurosci. 106, 623-633. doi: 10.1037/0735-7044. 106.4.623

Roesch, M. R., and Olson, C. R. (2003). Impact of expected reward on neuronal activity in prefrontal cortex, frontal and supplementary eye fields and premotor cortex. J. Neurophysiol. 90, 1766-1789. doi: 10.1152/jn.00 019.2003

Root, D. H., Melendez, R. I., Zaborszky, L., and Napier, T. C. (2015). The ventral pallidum: subregion-specific functional anatomy and roles in motivated behaviors. Prog. Neurobiol. 130, 29-70. doi: 10.1016/j.pneurobio.2015. 03.005

Rovó, Z., Ulbert, I., and Acsády, L. (2012). Drivers of the primate thalamus. J. Neurosci. 32, 17894-17908. doi: 10.1371/journal.pone.0247985

Saalmann, Y. B. (2014). Intralaminar and medial thalamic influence on cortical synchrony, information transmission and cognition. Front. Syst. Neurosci. 8:83. doi: $10.3389 /$ fnsys.2014.00083

Schaeffer, D. J., Hori, Y., Gilbert, K. M., Gati, J. S., Menon, R. S., and Everling, S. (2020). Divergence of rodent and primate medial frontal cortex functional connectivity. Proc. Natl. Acad. Sci. U S A 117, 21681-21689. doi: 10.1073/pnas. 2003181117

Schiff, N. D., and Purpura, K. P. (2002). Towards a neurophysiological foundation for cognitive neuromodulation through deep brain stimulation. Thal. Rel. Syst. 2, 55-69. doi: 10.1016/S1472-9288(02)00028-6

Schmitt, L. I., Wimmer, R. D., Nakajima, M., Happ, M., Mofakham, S., and Halassa, M. M. (2017). Thalamic amplification of cortical connectivity sustains attentional control. Nature 545, 219-223. doi: 10.1038/nature22073

Sesack, S. R., Deutch, A. Y., Roth, R. H., and Bunney, B. S. (1989). Topographical organization of the efferent projections of the medial prefrontal cortex in the rat. An anterograde tract-tracing study with Phaseolus vulgaris leucoagglutinin. J. Comp. Neurol. 290, 213-242. doi: 10.1002/cne.902900205

Sharpe, M. J., and Killcross, S. (2015). The prelimbic cortex uses higher-order cues to modulate both the acquisition and expression of conditioned fear. Front. Syst. Neurosci. 8:235. doi: 10.3389/fnsys.2014.00235

Shenoy, K. V., Sahani, M., and Churchland, M. M. (2013). Cortical control of arm movements: a dynamical systems perspective. Ann. Rev. Neurosci. 36, 337-359. doi: 10.1146/annurev-neuro-062111-150509

Sierveritz, B., García-Muñoz, M., and Arbuthnott, G. W. (2019). Thalamic afferents to prefrontal cortices from ventral motor nuclei in decision-making Eur. J. Neurosci. 49, 646-657. doi: 10.1111/ejn.14215

Skvortsova, V., Palminteri, S., and Pessiglione, M. (2014). Learning to minimize efforts versus maximizing rewards: computational principles and neural correlates. J. Neurosci. 34, 15621-15630. doi: 10.1523/JNEUROSCI.135014.2014

Sloan, H. L., Good, M., and Sunnett, S. B. (2006). Double dissociation between hippocampal and prefrontal lesions on an operant delayed matching task and a water maze reference memory task. Behav. Brain Res. 171, 116-126. doi: 10.1016/j.bbr.2006.03.030

Stephens, D. W., and Krebs, J. R. (1986). Foraging Theory. Princeton, NJ: Princeton University Press.

Stevens, A. A., and Mair, R. G. (1998). Auditory conditional discrimination deficits without delays in rats with lesions of either frontal cortex or medial thalamus. Psychobiology 26, 205-215.

Tanaka, M., and Kunimatsu, J. (2011). Contribution of the central thalamus to the generation of volitional saccades. Eur. J. Neurosci. 33, 2046-2057. doi: 10.1111/j.1460-9568.2011.07699.x

Tanibuchi, I., and Goldman-Rakic, P. S. (2003). Dissociation of spatial-, object-, and sound-coding neurons in the mediodorsal nucleus of the primate thalamus J. Neurophysiol. 89, 1067-1077. doi: 10.1152/jn.00207.2002

Tanibuchi, I., and Goldman-Rakic, P. S. (2005). Comparison of oculomotor neuronal activity in paralaminar and mediodorsal thalamus in the rhesus monkey. J. Neurophysiol. 93, 614-619. doi: 10.1152/jn.00969.2003 
Totah, N. K. B., Jackson, M. E., and Moghaddam, B. (2013). Preparatory attention relies on dynamic interactions between prelimbic cortex and anterior cingulate cortex. Cereb. Cortex 23, 729-738. doi: 10.1093/cercor/bhs057

Totah, N. K. B., Kim, Y. B., Homayoun, H., and Moghaddam, B. (2009). Anterior cingulate neurons represent errors and preparatory attention within the same behavioral sequence. J. Neurosci. 29, 6418-6426. doi: 10.1523/JNEUROSCI. 1142-09.2009

Uylings, H. B. M., Groenewegen, H. J., and Kolb, B. (2003). Do rats have a prefrontal cortex? Behav. Brain Res. 146, 3-17. doi: 10.1016/j.bbr.2003.09.028

Van der Werf, Y. D., Scheltens, P., Lindeboom, J., Witter, M. P., Uylings, H. B., and Jolles, J. (2003). Deficits of memory, executive functioning and attention following infarction in the thalamus; a study of 22 cases with localized lesions. Neuropsychologia 41, 1330-1344. doi: 10.1016/s0028-3932(03) 00059-9

Vertes, R. P. (2002). Analysis of projections from the medial prefrontal cortex to the thalamus in the rat, with emphasis on nucleus reuniens. J. Comp. Neurol. 442, 163-187. doi: 10.1002/cne.10083

Vertes, R. P. (2004). Differential projections of the infralimbic and prelimbic cortex in the rat. Synapse 51, 32-58. doi: 10.1002/syn.10279

Vertes, R. P., Hoover, W. B., Do Valle, A. C., Sherman, A., and Rodriguez, J. J. (2006). Efferent projections of reunions and rhomboid nuclei of the thalamus in the rat. J. Comp. Neurol. 499, 768-796. doi: 10.1002/cne.21135

Vertes, R. P., Linley, S. B., and Hoover, W. B. (2015). Limbic circuitry of the midline thalamus. Neurosci. Biobehav. Rev. 54, 89-107. doi: 10.1016/j. neubiorev.2015.01.014

Vertes, R. P., and Hoover, W. B. (2008). Projections of the paraventricular and paratenial nuclei of the dorsal midline thalamus in the rat. J. Comp. Neurol. 508, 212-237. doi: 10.1002/cne.21679

Victor, M., Adams, R. D., and Collins, G. H. (1971). The Wernicke-Korsakoff syndrome. A clinical and pathological study of 245 patients, 82 with post-mortem examinations. Contemp. Neurol. Ser. 7, 1-206.

Vogt, B. A., Hof, P. R., Zilles, K., Vogt, L. J., Herold, C., and PalomeroGallagher, N. (2013). Cingulate area 32 homologies in mouse, rat, macaque and human: cytoarchitecture and receptor architecture. J. Comp. Neurol. 521, 4189-4204. doi: 10.1002/cne.23409

von Cramon, D. Y., Hebel, N., and Schuri, U. (1985). A contribution to the anatomical basis of thalamic amnesia. Brain 108, 993-1008. doi: 10.1093/brain/108.4.993

Walton, M. E., Kennerley, S. W., Bannerman, D. M., Phillips, P. E. M., and Rushworth, M. F. S. (2006). Weighing up the benefits of work: behavioral and neural analyses of effort-related decision making. Neural Netw. 19, 1302-1314. doi: 10.1016/j.neunet.2006.03.005

Warburton, E. C., Baird, A. L., and Aggleton, J. P. (1997). Assessing the magnitude of the allocentric spatial deficit associated with complete loss of the anterior thalamic nuclei in rats. Behav. Brain Res. 87, 223-232. doi: 10.1016/s01664328(97)02285-7

Watanabe, Y., and Funahashi, S. (2004a). Neuronal activity throughout the primate mediodorsal nucleus of the thalamus during oculomotor delayedresponses: I. Cue-, delay-, and response-period activity. J. Neurophysiol. 92, 1738-1755. doi: 10.1152/jn.00994.2003

Watanabe, Y., and Funahashi, S. (2004b). Neuronal activity throughout the primate mediodorsal nucleus of the thalamus during oculomotor delayedresponses. II. Activity encoding visual versus motor signal. J. Neurophysiol. 92, 1756-1769. doi: 10.1152/jn.00995.2003

Watanabe, Y., and Funahashi, S. (2012). Thalamic mediodorsal nucleus and working memory. Neurosci. Biobehav. Rev. 36, 134-142. doi: 10.1016/j. neubiorev.2011.05.003

Whishaw, I. Q., and Dringenberg, H. C. (1991). How does the rat (Rattus norvegicus) adjust food-carrying responses to the influences of distance, effort, predatory odor, food size and food availability? Psychobiology 19, 251-261.

Whishaw, I. Q., Dringenberg, H. C., and Comery, T. A. (1992). Rats (Rattus norvegicus) modulate eating speed and vigilance to optimize food consumption: effects of cover, circadian rhythm, food deprivation, and individual differences. J. Comp. Psychol. 106, 411-419. doi: 10.1037/0735-7036.106.4.411

Winocur, G., and Eskes, G. (1998). Prefrontal cortex and caudate nucleus in conditional associative learning: dissociated effects of selective brain lesions in rats. Behav. Neurosci. 112, 89-101. doi: 10.1037/0735-7044. 112.1 .89

Wolff, M., Gibb, S., Cassel, J.-C., and Dalrymple-Alford, J. C. (2008). Anterior but not intralaminar thalamic nuclei support allocentric spatial memory. Neurobiol. Learn. Mem. 90, 71-80. doi: 10.1016/j.nlm.2008.01.007

Wolff, M., and Vann, S. D. (2019). The cognitive thalamus as a gateway to mental representations. J. Neurosci. 39, 3-14. doi: 10.1523/JNEUROSCI.047918.2018

Wyder, M. T., Massoglia, D. P., and Stanford, T. R. (2003). Quantitative assessment of the timing and tuning of visual-related, saccade-related, and delay period activity in primate central thalamus. J. Neurophysiol. 90, 2029-2052. doi: 10.1152/jn.00064.2003

Xiao, D., Zikopoulos, B., and Barbas, H. (2009). Laminar and modular organization of prefrontal projections to multiple thalamic nuclei. Neuroscience 161, 1067-1081. doi: 10.1016/j.neuroscience.2009.04.034

$\mathrm{Xu}, \mathrm{Y}$. (2018). The posterior parietal cortex in adaptive visual processing. Trends Neurosci. 41, 806-822. doi: 10.1016/j.tins.2018.07.012

Yin, A., Tseng, P. H., Rajangam, S., Lebedev, M. A., and Nicolelis, M. A. L. (2018). Place cell-like activity in the primary sensorimotor and premotor cortex during monkey whole-body navigation. Sci. Rep. 8:9184. doi: 10.1038/s41598018-27472-4

Young, H. L., Stevens, A. A., Converse, E., and Mair, R. G. (1996). A comparison of temporal decay in place memory tasks in rats (Rattus norvegicus) with lesions affecting thalamus, frontal cortex, or the hippocampal system. Behav. Neurosci. 110, 1244-1260. doi: 10.1037/0735-7044.110.6.1244

Zhang, Y., Bailey, K. R., Toupin, M. M., and Mair, R. G. (2005). Involvement of ventral pallidum in prefrontal cortex-dependent aspects of spatial working memory. Behav. Neurosci. 119, 399-409. doi: 10.1037/0735-7044.119. 2.399

Zhang, Y., Burk, J. A., Glode, B. M., and Mair, R. G. (1998). Effects of thalamic and olfactory cortical lesions on continuous olfactory delayed nonmatchingto-sample and olfactory discrimination in rats (Rattus norvegicus). Behav. Neurosci. 112, 39-53. doi: 10.1037/0735-7044.112.1.39

Zhu, Y., Nachtrab, G., Keyes, P., Allen, W. E., Luo, L., and Chen, X. (2018). Dynamic salience processing in paraventricular thalamus gates associative learning. Science 362, 423-429. doi: 10.1126/science.aat0481

Zola-Morgan, S., and Squire, L. R. (1985). Amnesia in monkeys after lesions of the mediodorsal nucleus of the thalamus. Ann. Neurol. 17, 558-564. doi: 10.1002/ana.410170605

Conflict of Interest: The authors declare that the research was conducted in the absence of any commercial or financial relationships that could be construed as a potential conflict of interest.

Copyright 2021 Mair, Francoeur and Gibson. This is an open-access article distributed under the terms of the Creative Commons Attribution License (CC BY). The use, distribution or reproduction in other forums is permitted, provided the original author(s) and the copyright owner(s) are credited and that the original publication in this journal is cited, in accordance with accepted academic practice. No use, distribution or reproduction is permitted which does not comply with these terms. 